\title{
WestVirginiaUniversity
}

THE RESEARCH REPOSITORY @ WVU

Graduate Theses, Dissertations, and Problem Reports

2018

\section{Ex-Vivo Model for Muscle Exposure to Tumor Cell Conditioned \\ Media}

Ikttesh K. Chahal

Follow this and additional works at: https://researchrepository.wvu.edu/etd

\section{Recommended Citation}

Chahal, Ikttesh K., "Ex-Vivo Model for Muscle Exposure to Tumor Cell Conditioned Media" (2018).

Graduate Theses, Dissertations, and Problem Reports. 5330.

https://researchrepository.wvu.edu/etd/5330

This Thesis is protected by copyright and/or related rights. It has been brought to you by the The Research Repository @ WVU with permission from the rights-holder(s). You are free to use this Thesis in any way that is permitted by the copyright and related rights legislation that applies to your use. For other uses you must obtain permission from the rights-holder(s) directly, unless additional rights are indicated by a Creative Commons license in the record and/ or on the work itself. This Thesis has been accepted for inclusion in WVU Graduate Theses, Dissertations, and Problem Reports collection by an authorized administrator of The Research Repository @ WVU. For more information, please contact researchrepository@mail.wvu.edu. 


\title{
Ex-Vivo Model for Muscle Exposure to Tumor Cell Conditioned Media
}

\author{
Ikttesh K. Chahal \\ Thesis Submitted \\ to the School of Medicine \\ at West Virginia University \\ in partial fulfillment of the requirements for the degree of \\ Master of Science in \\ Exercise Physiology
}

Emidio E. Pistilli, Ph.D., Chair

Elena N. Pugacheva, Ph.D.

Stephen E. Alway, Ph.D.

Junaith S. Mohamed, Ph.D.

Department of Exercise Physiology

Morgantown, West Virginia

2018

Keywords: Cancer Cachexia, Skeletal Muscle, Interleukin-15, JAK/STAT pathway, muscle fatigue

Copyright 2018 Ikttesh K. Chahal 


\section{ABSTRACT \\ Ex-Vivo Model for Muscle Exposure to Tumor Cell Conditioned Media}

\section{Ikttesh K. Chahal}

Cancer-induced cachexia is a severe condition resulting in muscle wasting, muscle dysfunction, and an overall increased morbidity and mortality. The cachectic condition is often attributed to altered metabolism, aberrant activation of signaling pathways, and changes in the microenvironment of the skeletal muscle (SKM) through exposure to various host-derived and tumor-derived paracrine factors, delivered through the blood circulation. Previous data from our lab has shown that an increased signaling of Interleukin-15 (IL-15) results in enhanced oxidative capacity, promotion of a more oxidative phenotype and attenuation of muscle fatigue. This effect of IL-15 has yet to be characterized in a cancer-induced model of muscle dysfunction. Therefore, through this project, we sought to establish an ex-vivo working model for muscle exposure to tumor cell conditioned media using an IL-15TG overexpressing IL-15 mouse model; thereby allowing to test IL-15's role in the JAK/STAT signaling pathway, an oncogenic signaling cascade that exerts tumor-intrinsic and tumor-extrinsic effects. Current studies investigating the role of tumor secreted factors on muscle function in-vitro do so by exposing muscle cells to solutions containing tumor cell conditioned media. However, this is an incomplete representation and assessment of the in-vivo system. In our improved model, the whole extensor digitorum longus (EDL) muscles are used in combination with human breast cancer cell line conditioned media (CM). We hypothesized that the cancer cell-secreted factors will induce muscle dysfunction and affect mediators of the JAK/STAT pathway in comparison to control unconditioned media (UCM). Our initial analyses using the EDL muscles from WT mice, IL15TG littermate control mice, and IL-15TG overexpressing IL-15 mice revealed no significant differences in the functional output in either CM or UCM conditions. However, recent literature suggests that at least a 2 hour pre-incubation period is needed to see analogous effects seen with a shared circulation of cancer cell-secreted factors and muscle cells in-vivo. Additional experiments using an extended exposure are needed to improve the translational potential of the proposed model. However, our western blot analyses revealed a statistical significance $(\mathrm{p}<0.05)$ in the phosphorylated STAT3 (pSTAT3) in the stimulated CM compared to the stimulated UCM conditions in the TG group. This finding is supported in the literature and suggests that samples that undergo stimulation and are exposed to CM show a higher phosphorylation of STAT3. It also validates the overexpressing properties of the IL-15TG mouse since IL-15 binding is known to induce and activate the JAK3/STAT3 pathway. In conclusion, this research provides support for further development of this ex-vivo muscle exposure to tumor cell conditioned media model. 


\section{Ex-Vivo Model for Muscle Exposure to Tumor Cell Conditioned Media}

Ikttesh K. Chahal

Division of Exercise Physiology, West Virginia University School of Medicine.

CHAPTER 1: Introduction $\quad 1$

CHAPTER 2: Literature Review $\quad 5$

2.1 Cancer Cachexia $\quad 5$

2.2 Models of Cancer Cachexia $\quad 7$

2.3 Role of IL-15 9

2.4 JAK/STAT pathway 11

2.5 Reactive Oxygen Species (ROS) and Cancer 13

2.6 Background Summary and Significance 15

CHAPTER 3: Study Design and Methods $\quad 16$

3.1 Study Design Overview 16

3.2 Mice Models $\quad 17$

3.3 Animal Care 17

3.4 Media Collection $\quad 18$

$\begin{array}{llr}\text { 3.5 } & \text { Stock Preparation } & 18\end{array}$

3.6 Mouse Surgery 19

3.7 Muscle Function Analysis of EDL muscle in C57Bl/6J WT mice, IL-15TG littermate control and IL-15TG overexpressing IL-15 mice.

3.8 pH Measurement 22

3.9 Protein Quantification $\quad 22$

3.10 Western Blot $\quad 22$

3.11 Protein Carbonyl Assay 23

3.12 Data Analysis $\quad 24$

CHAPTER 4: Results $\quad 24$

4.1 Evaluation of EDL Muscle Function using Different Conditions 24

4.2 Determination of Conditioned Media Percentage 27

4.3 Effect of $25 \%$ diluted CM on Contractile Data of muscles exposed to cancer cell-secreted factors from the MDA-MB-231 cell line 28

4.4 Protein Carbonyl Concentrations to measure for Oxidative Stress 33

4.5 Measurement of $\mathrm{pH}$ changes $\quad 34$

4.6 Effect of $25 \%$ diluted CM on Contractile Data of muscles following 2-hour Extended Exposure

4.7 Western Blot Analysis of pSTAT3 and total STAT3 36

CHAPTER 5: Discussion $\quad 39$

CHAPTER 6: References $\quad 45$

CHAPTER 7: Supplementary Data $\quad 54$ 


\title{
Ex-Vivo Model for Muscle Exposure to Tumor Cell Conditioned Media
}

\author{
Ikttesh K. Chahal \\ Division of Exercise Physiology, West Virginia University School of Medicine
}

\section{CHAPTER 1.}

\section{Introduction}

Cancer-associated muscle weakness is a well-observed syndrome for which there is currently no treatment. Muscle weakness can be caused by reduced muscle mass or reduced muscle function and in advanced diseases, including cancer, it is likely due to a combination of both. Aside from a significantly decreased quality of life, the overall prognosis associated with cancer cachexia, which is defined as muscle wasting and muscle dysfunction, accounts for approximately $40-50 \%$ of cancer patient morbidity [1]. Although cachexia is considered as an important factor associated with cancer-related deaths, no experimental link has been determined to show the exact mechanism by which this syndrome arises [2]. The literature proposes that the main contributors of cachexia are abnormal fluctuations in cytokines, hormones and tumorderived factors that alter the cellular environment and thereby exacerbate cachectic symptoms [1]. This may be due in part to a reduction of mitochondrial function in cancer cachexia leading to loss of muscle function. Studying the effects of the altered biomarkers, signaling pathways, and their subsequent effect on gene expression is a potential avenue to further understand the mechanisms involved in cancer cachexia. Previous work from our lab suggests that increasing signaling of the cytokine Interleukin-15 (IL-15) increases mitochondrial mass, and enhances oxidative capacity in skeletal muscle [3, 4]. However, we do not know if the effect of IL-15 will improve these conditions in a cancer-induced model of muscle dysfunction. 
A second potential mediator of muscle atrophy seen in cancer cachexia may be via the Janus kinase and signal transducers and activators of transcription (JAK/STAT) signaling pathway as it is an important oncogenic signaling cascade that exerts tumor-intrinsic and tumorextrinsic effects, supporting tumor survival and metastasis [5]. Inhibition of the JAK/STAT pathway has been shown to reduce cancer hallmarks such as proliferation, survival, angiogenesis, and tumor metabolism [6]. Once activated, JAK proteins phosphorylate tyrosine residues present in the cytoplasmic region of the receptor, thereby creating binding sites for STATs [7]. These STAT proteins form dimers, allowing them to be further activated through phosphorylation. These phosphorylated dimers termed pSTAT translocate to the nucleus and bind specific promoter sequences to modulate gene transcription of cellular processes involving proliferation, differentiation and apoptosis [7]. Further, activation of pSTAT3 has also been reported to induce the activation of two different proteolytic pathways in the muscle - caspase 3, and the MAFbx/Atrogin-1 and MuRF-1 pathway [8]. Our preliminary data shows a potential role for the JAK/STAT signaling pathway downstream of IL-15 binding, impacting muscle function and mass in cancer cachexia. Therefore, it is expected that through this project we will be able to use a model that links muscle function and signaling pathways while closely mimicking conditions that are seen in vivo.

Our central aim was to evaluate JAK/STAT IL-15 regulation in cancer cachexia using an ex-vivo model that can emulate a system seen in tumor-bearing animals. In this model, a whole muscle is constantly exposed to tumor-produced factors via incubation in conditioned media used to culture a human breast cancer cell line-MDA-MB-231. We measured the expression levels and associated phosphorylation levels of certain mediators in the JAK/STAT pathway (such as STAT3 and pSTAT3) that directly affect gene expression. In doing so, we determined a 
link between the functional properties of a whole muscle and mediators of the pathway downstream of IL-15 binding. Traditionally, the effects of conditioned media have been observed in individual cells such as myocytes [8,9]. However, the effects have not been reported on a functional muscle since the development of an external environment with minimal alteration to natural conditions has not yet been achieved. Another key component of this project was that the contractile data was analyzed from the whole muscle, which permitted us to have a functional output, contributing to the novelty of this project. In this research, we used two different mouse models, including wild type mice (WT; strain C57BL/6J) and transgenic mice (IL-15TG - over expressers of IL-15). These mouse models allowed us to build on the results of the preliminary data and previously published work by our laboratory. We expected that the protein levels of the mediators downstream of IL-15 binding through the JAK/STAT pathway such as phosphorylation of STAT3 would be elevated in the overexpressing IL-15 mouse model in comparison to their littermate controls. We also expected to see similar trends with the muscle function of the IL-15TG mice as the pro-oxidative state and elevated fatigue resistance phenotype may in fact aid in decreasing symptoms associated with cancer cachexia.

\section{Specific Aim 1: Determine the effects of conditioned media used to grow cancer cells on $e x$ -} vivo muscle function.

Our working hypothesis is that $25 \%$ diluted conditioned media will reduce ex vivo muscle function causing reduced contractile forces when compared to the muscle function using control media ( $25 \%$ diluted unconditioned media). From our preliminary data, we know that tumors produced by orthotopic injection of EO771 murine mammary gland cancer cell line into syngeneic C57BL/6J mice cause muscle dysfunction and muscle wasting. However, we do not 
know if it is the cancer cell-derived factors that are causing this muscle fatigue and muscle wasting. Therefore, we want to evaluate this effect using an ex-vivo whole muscle model where individual components, muscles, tumor cells, or tumor cell-derived factors could be evaluated. We expect to see a decrease in muscle force output in the muscles exposed to $25 \%$ diluted conditioned media from the MDA-MB-231 human breast cancer cell line in comparison to $25 \%$ diluted unconditioned media. Similarly, the skeletal muscle from IL-15TG mouse is expected to demonstrate a greater preservation of muscle force.

Specific Aim 2: Determine the effects of $25 \%$ diluted conditioned media on the mediators in the JAK3/STAT3 signaling pathway in an ex vivo model.

Our working hypothesis is that cancer cell-derived factors in conditioned media will have an effect on JAK3/STAT3 pathway activation. From our preliminary data, we observed increased mRNA expression of both, Murf-1 and Atrogin-1, markers of muscle atrophy, when exposed to conditioned media from EO771 cells, a cultured murine cancer cell line. However, effects of cancer cell-derived factors on the phosphorylation of proteins such as JAK3, STAT3, STAT5 had not been determined prior to our study. Western blot analyses will be used to measure expression level of JAK/STAT proteins and their phosphorylation in protein homogenates obtained from mice extensor digitorum longus (EDL) muscles exposed to $25 \%$ diluted conditioned media, in comparison to control protein homogenates of mice EDLs exposed to $25 \%$ diluted unconditioned media. 


\section{CHAPTER 2.}

\section{Literature Review}

\subsection{Cancer Cachexia}

Cancer is a major public health problem worldwide, with an estimated 14.1 million new cases of cancer diagnoses annually [10]. In 2017, the American Cancer Society (ACS) estimated that in the United States, over 600,000 deaths are due to cancer yearly; making it the second leading cause of death [10]. Cancer-induced cachexia, a syndrome characterized by loss of muscle mass, fatigue and weakness is experienced by almost fifty percent of all cancer patients [11]. A greater prevalence is observed in patients with advanced stage cancers, with almost eighty percent of patients presenting with this severe wasting syndrome [1, 12]. Despite being considered as an emerging field of research, the molecular mechanisms leading to skeletal muscle loss with cachexia are still largely unknown. Currently, there are no approved drug therapies or effective medical interventions that completely reverse cancer cachexia [13].

Cancer cachexia can be characterized by loss or extreme atrophy of skeletal muscle mass that may or may not be accompanied by loss of fat mass [10]. Another key component of cancer cachexia is severe fatigue in patients, which is commonly credited to the loss of muscle mass [2]. However, it has been shown that cancer-induced muscle fatigue can occur without loss of muscle mass [1]. Figure 1 represents the common presentations seen in cachexia that include inflammation, anorexia and hormonal aberrations. Changes in the microenvironment of the skeletal muscle through exposure to various host-derived and tumor-derived paracrine factors delivered through the blood circulation are now being studied and attributed as possible causes of 
this disease [1], [14]. Specifically, evidence suggests that markers of inflammation and biomarkers contributing to protein degradation can contribute to cachectic symptoms $[15,16]$.

In particular, studies evaluating the metabolic alterations leading to cancer cachexia have revealed pathways including the NF-kB (nuclear factor kappa-light-chain-enhancer of activated B cells) and JAK/STAT pathway [17]. Similarly, the ubiquitin-proteasome system (UPS) pathway has also been shown to play a key role in muscle protein breakdown, especially through activation of its main regulators - ubiquitin E3 ligases, muscle ring finger protein 1 (MuRF-1), and atrophy gene 1/muscle atrophy F-box (Atrogin-1/MAFbx) [16-19]. Further investigation is needed in studying the different mediators of these signaling pathways as they can serve as potential therapeutic opportunities. In fact, a first-in-human outpatient phase I dose escalation trial of subcutaneous (SC) recombinant human IL-15 (rhIL-15) was recently conducted to study the effectiveness and safety of rhIL-15 in treating refractory solid tumors in cancer patients [20]. IL-15, being a powerful immunostimulatory cytokine, is involved in the proliferation, differentiation, and activation of NK cells and CD8 $+\mathrm{T}$ cells, and actually utilizes certain JAK/STAT proteins as a means of initiating signal transduction for cellular activation [21]. 


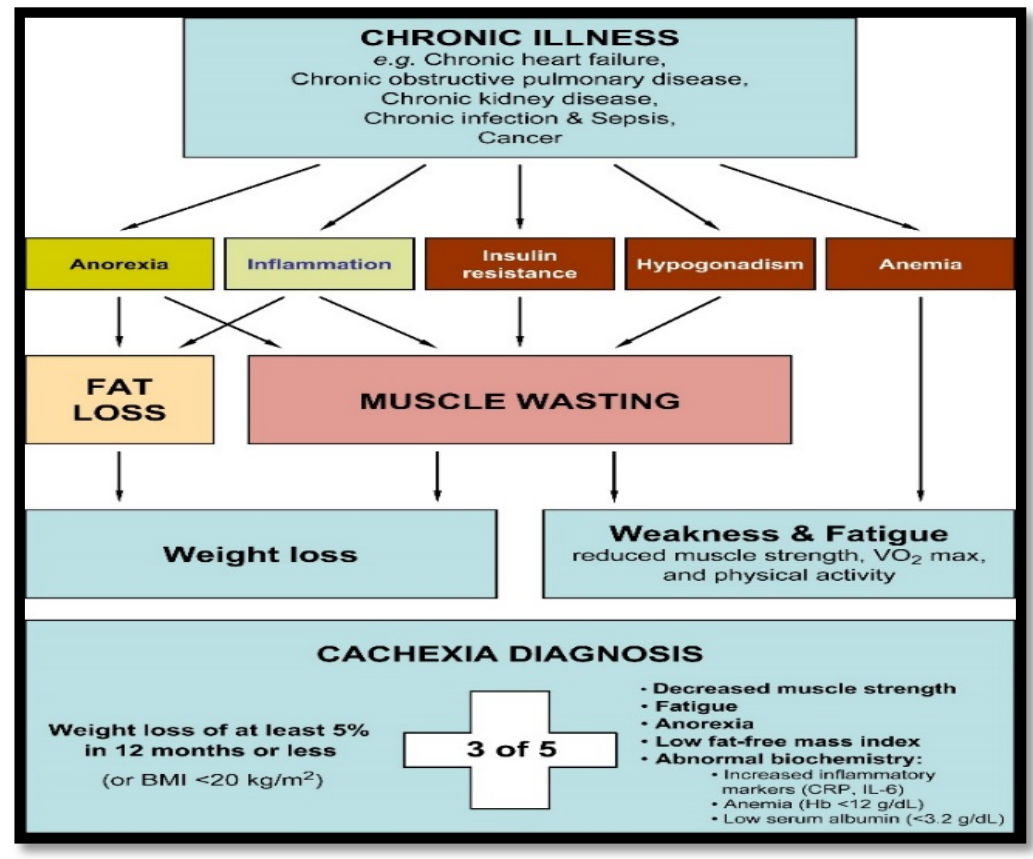

Figure 1: Conceptual definition of cachexia through a schematic.

Above image from Evans et al. [15] represents the conceptual definition of cachexia seen in a chronic disease. Inflammation, anorexia, and hormonal aberrations are common presentations.

\subsection{Models of Cancer Cachexia}

Despite the lack of approved therapies or treatments for cancer cachexia, multiple models of cancer cachexia are being used to gain insight into the mechanisms behind muscle wasting, as well as potential therapeutic targets [22]. Many studies are employing the use of animal models; by either genetically modifying the animals or through tumor implantation, to understand these underlying mechanisms. Since cytokines, myokines, and other factors also contribute to muscle mass regulation, cell culture models are also used to study signaling pathways and their responses. There are many benefits to using cultured cell lines in cancer research; a major benefit is that cell lines self-replicate and supply a relatively homogeneous cell population using a standard cell culture medium [23]. One of the most widely used breast cancer cell lines is MDA- 
MB-231, a metastatic mammary carcinoma cell line that was established in 1973 at M.D Anderson Cancer Center [24, 25]. This human cell line is typically used as a model for estrogen receptornegative breast cancers and is highly aggressive both in vitro and in vivo [26, 27]. Therefore, MDA-MB-231 is a valuable cell culture model to study cancer cachexia due to its invasive and metastatic properties.

Currently, different groups are using such models to study different cancer types and their presentations. Puppa et al. [28] reported a 16\% skeletal muscle attenuation in gp130-KO mice in comparison to wild-type mice, in which both of the groups were subjected to Lewis lung carcinoma (LLC) cells. Glycoprotein 130 (gp130) is a common signal transducer that typically activates STAT3, an intracellular transcription factor implicated in stimulating protein muscle loss $[8,29]$. They reported decreases in LLC-induced phosphorylation of muscle STAT3 and FOXO3, a tumor suppressor gene $[28,30]$. Through in vitro experiments using C2C12 myotubes, they reported a repression in LLC-induced atrophy signaling [28]. However, they concluded that further development of this cachexia model is needed since the inhibition of skmgp130 was not sufficient to suppress the LLC-induced signaling of atrophic pathways [28]. Investigators using the mouse $\mathrm{C} 26$ colon carcinoma cancer model treated $\mathrm{C} 2 \mathrm{C} 12$ myotubes with conditioned medium from $\mathrm{C} 26$ cancer cells to observe the signaling pathways activated in this model of induced atrophy [31]. They reported an increase in STAT activation through STAT3 phosphorylation and myotube atrophy induced by C26 conditioned media [31]. Another group using the C26 model reported similar mouse myotube atrophy using conditioned media by measuring myotube diameter [9]. However, this response is less in vitro when compared to in vivo muscles of mice bearing C26 tumors [9]. Using transcriptomic and metabolomic profiling, Fukawa et al. [32] observed impaired muscle growth, increased oxidative stress through 
excessive fatty acid oxidation in human myotubes, suggesting the importance of experiments using myotubes to study the different atrophy-inducing conditions. These studies propose conditions analogous to those in-vivo as they are all trying to stimulate an environment representing shared circulation between cancer cells, their secreted factors, and muscle cells.

\subsection{Role of IL-15}

Gene and protein markers associated with cancer-induced loss of body weight, increased inflammation, and skeletal muscle protein breakdown show that pathways of fatigue often accompany cancer cachexia [15]. One such biomarker is IL-15, which is a recently discovered four-helix bundle cytokine [4]. Commonly, IL-15 has been investigated concerning its role in inflammation and its regulation in immune homeostasis [33]. It has been regarded as both pro-inflammatory and anti-inflammatory through its activation of Natural killer (NK) cells that display an increased immune response to invading pathogens [33, 34]. IL-15 has been seen as a potent inflammatory chemokine with potential in preventing and/or treating obesity and other metabolic disorders $[35,36]$.

Previous data collected in our laboratory using a transgenic mouse model lacking the gene for IL-15R $\alpha$ suggest that IL-15 promotes mitochondrial biogenesis [3]. IL-15R $\alpha$ is a highly specific non-signaling binding receptor for IL-15 and is thereby one of its primary regulators. Upon translation, the IL-15 gene encodes for two distinct isoforms of IL-15 including, a short 21-amino acid and a long 48 amino acid signal peptide [37]. It is the long IL-15 isoform that binds to IL-15R $\alpha$, allowing for the secretion of the cytokine-receptor complex from skeletal muscle [37]. However, even though free IL-15 secretion from the cell occurs, the binding between IL-15 and its receptor IL-15R $\alpha$ promotes an elevated ability for subsequent IL-15 signal 
transduction [38].

The use of a transgenic mouse model, that specifically overexpresses IL-15, has been shown to display an enhanced endurance capacity and to increase the expression of mitochondrial associated factors and mitochondrial mass [3, 4, 39]. An increased expression of myosin heavy chain (MHC-1) and troponin 1 mRNA isoform expression in the muscle tissue of the IL-15 transgenic mice is credited to this oxidative phenotype [39]. Further, Quinn et al. [35] reported increased myofibrillar protein accumulation in IL-15 overexpressing myogenic cells, along with hypertrophied myotube morphology. These data provide evidence for the positive role of IL-15 in facilitating an elevated substrate utilization capacity in skeletal muscle through its action in different molecular pathways $[36,37,40]$. However, there is still no clear distinction of the molecular mediators responsible for these effects. Thus, there is a need to further study the different signaling pathways that are downstream of IL-15 action, with some of them being shown in Figure 2. 


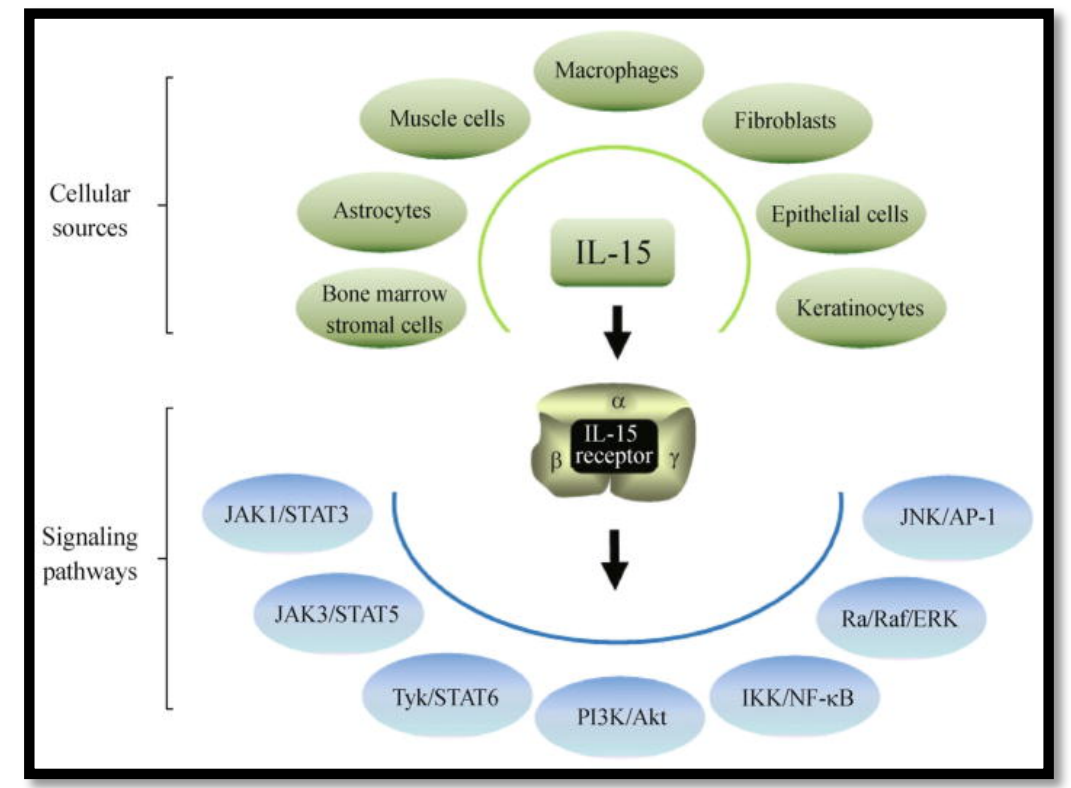

Figure 2: Sources of IL-15 and Signaling Pathways implicated with IL-15.

Above image from Ye [41] depicts the multiple cellular sources and signaling pathways of IL-15 to illustrate the diverse functions of $I L-15$.

\subsection{JAK/STAT pathway}

The Janus kinase activation of signal transducer and activator of transcription proteins (JAK/STAT) signaling pathway is one of the most commonly studied pathways that is downstream of IL-15 action $[12,41]$. The JAK/STAT pathway is a simple membrane-to-nucleus pathway whereby extracellular factors control gene expression (Figure 3) [42]. Activation of the receptorassociated JAKs occurs with the binding of the ligand, causing auto-phosphorylation on the intracellular tails, thereby making docking sites for the cytoplasmic transcription factors, STATs, to bind and become phosphorylated [42]. Activated STAT directly binds to DNA and has the ability to regulate gene expression $[43,44]$. Overall, the JAK/STAT signaling pathway has been 
found to occur in virtually every tissue of the body, giving it the ability to effect energy

metabolism in many cell types due to its large number of intracellular functions [45].

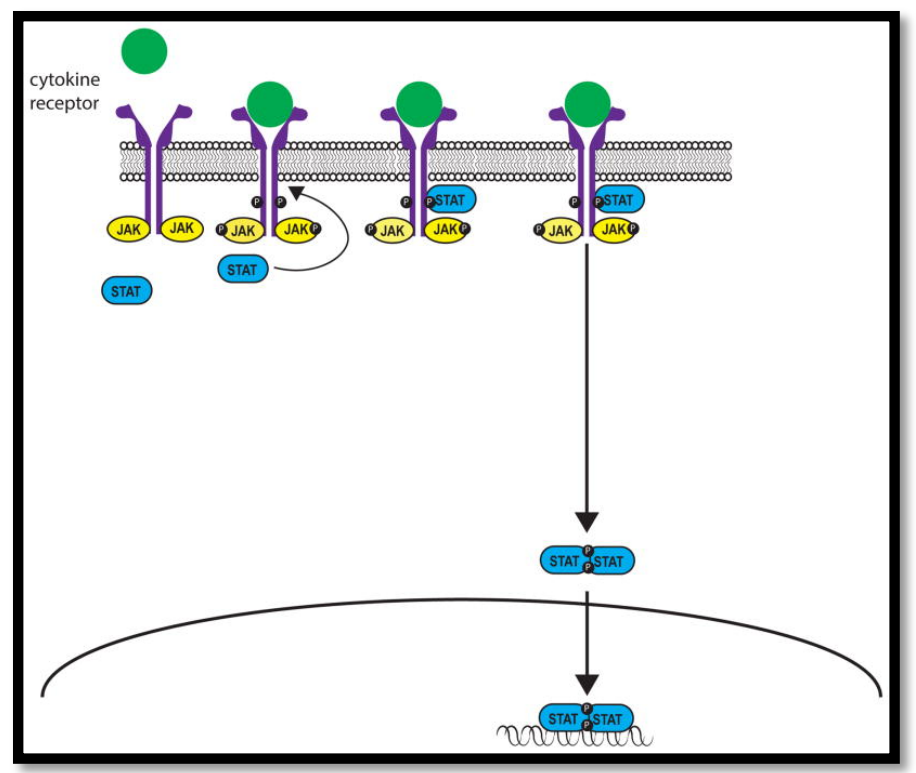

Figure 3: Mechanism of JAK/STAT Pathway.

The figure above from O'Shea et al. [44] shows the mechanism by which the JAK/STAT signaling pathway plays a role in gene expression through its communication that starts from transmembrane receptors to the nucleus.

The JAK/STAT signaling pathway has multiple mediators and isoforms that correspond to the distinct in vivo roles of this cascade. There are four JAKs: JAK1, JAK2, JAK3, and TYK2, which can selectively bind various receptor chains, while seven mammalian STAT family members, namely STAT1, STAT2, STAT3, STAT4, STAT5a, STAT5b, and STAT6 have been identified [42]. Specific to IL-15, the mechanism begins with the binding of the IL-15 to the IL-15 receptor complex, which cause the auto-phosphorylation of the JAK isoforms (JAK1 and/or JAK3), further inducing the phosphorylation of STAT3 and/or STAT5 [41]. Since different cytokines have the ability to activate different STATs, the underlying mechanisms by which gene transcription is affected can be explained using new technologies that determine transcription factor binding, genome wide assessments, and using knockout mice models. 
Signaling through STAT3 has been reported to increase the acute phase response gene expression in the liver [46]. The acute phase response is a generalized response of the innate immune system, consisting of a series of specific physiological reactions caused by the upregulation (up to several thousand-fold) of some 40 genes upon stimulation [47, 48]. Elevations in acute phase response proteins in the serum have been linked to cancer cachexia [49-51]. Further, Silva et al. reported that the activation of pSTAT3 in $\mathrm{C} 2 \mathrm{C} 12$ myotubes induces the activation of two different proteolytic pathways in the muscle - caspase 3, and the MAFbx/Atrogin-1 and MuRF-1 pathways [8]. Finally, STAT3 activation has also been reported to enhance tumorigenesis, metastasis, and immune system suppression, factors all leading to its link to cancer cachexia [52].

\subsection{Reactive Oxygen Species (ROS) and Cancer}

Reactive oxygen species (ROS), are radicals, molecules or ions that exhibit a single unpaired electron in their outermost shell of electrons and therefore, are highly reactive [53]. ROS can be generated endogenously during mitochondrial oxidative metabolism through leaking of electrons as they pass through the different mitochondrial complexes of the electron transport chain (Figure 4A) [54]. Along with mitochondria, another major site of endogenous ROS generation are peroxisomes [55]. However, ROS production is also common through interactions within exogenous sources in the body such as cytokines, xenobiotic, and bacterial compounds [54].

An increase in ROS levels combined with a decrease in the cells natural antioxidant machinery leads to oxidative stress which results in the detrimental damage of proteins, nucleic acids, and lipids by ROS (Figure 4B) [54, 56, 57]. Single-stranded and double-stranded DNA breaks can be evoked through the reaction of ROS with nucleic acids causing a disruption 
between the nitrogenous and sugar phosphate backbones [58]. In the case of lipids, the reaction between ROS and polyunsaturated fatty acids can induce lipid peroxidation which can lead to alteration of the membrane by-layers [59]. Free radicals have also been reported to cause various forms of oxidative modifications in proteins [60]. Protein carbonylation is one such modification, which has been shown to occur commonly at the side chains of four distinct amino acids proline, lysine, threonine, and arginine [60]. This irreversible modification is clinically relevant since this carbonylation leads to the tagging of proteins for proteasomal degradation, and is thus considered one of the major hallmarks of oxidative stress-related disorders [60, 61]

ROS participation is seen in multiple pathological conditions including malignancies, diabetes, atherosclerosis, aging, and neurological degenerative diseases [54, 62, 63]. Elevated levels of ROS are seen in mostly all of the different types of cancers and have shown to promote pro-tumorigenic signaling, cancer cell proliferation, and tumor development and progression [64]. However, ROS have now been implicated to play a dual role in cancer and have also been shown to promote anti-tumorigenic signaling leading to oxidative stress-induced cancer cell death [65]. To study the mechanisms, in vitro studies use different types of ROS to observe and study the many aspects of ROS activity. Hydrogen peroxide $\left(\mathrm{H}_{2} \mathrm{O}_{2}\right)$ is a widely used ROS that induces oxidative stress in cells and Li et al. investigated the effects of IL-15 as a potential protective mechanism against this $\mathrm{H}_{2} \mathrm{O}_{2}$ mediated oxidative stress in $\mathrm{C} 2 \mathrm{C} 12$ myoblasts [66]. Interestingly, it was found that preincubation with IL-15 reduced the activities of intracellular creatine kinase and lactate dehydrogenase, enzymes involved in various metabolic pathways, thereby decreasing the ROS overload [66]. They also reported an upregulated mRNA expression level of uncoupling protein 3 (UCP3) and IL-15, eluding to enhanced protection of the mitochondrial networks. 


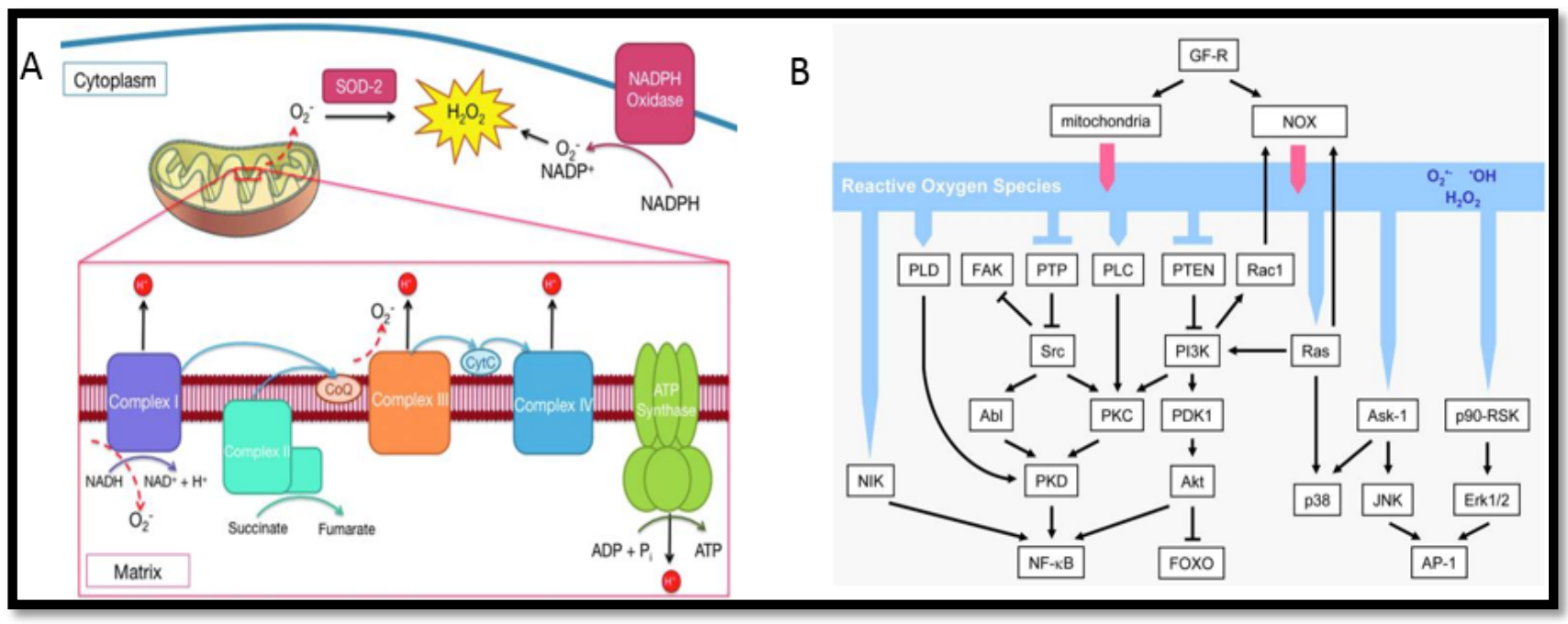

Figure 4: Souces of ROS production and Signaling Pathways implicated with ROS. Above images from Liang et al [56] show the different intracellular sources of ROS and the signaling pathways they affect. Panel $\boldsymbol{A}$ shows that the greatest amount of ROS production occurs at complexes I and III of the electron transport chain during oxidative phosphorylation. An additional source of intracellular $\mathrm{ROS}\left(\mathrm{O}_{2}^{-}\right)$is NADPH oxidase, shown at the top right of panel A. Panel B shows the different signaling cascades that ROS can induce that lead to damage of proteins, nucleic acids, and lipids.

\subsection{Background Summary and Significance}

Taken as a whole, the current literature is not able to provide the direct mechanism through which cancer cachexia takes place. While a wide variety of pathways have been implicated to play a role in muscle protein degradation and inhibition of muscle growth, the effects of IL-15, a myokine, have yet to be fully investigated. It is expected that this research will provide a better understanding of the changes in the JAK3/STAT3 signaling pathway that is downstream of IL-15 binding. The use of an IL-15TG mouse model that overexpresses IL-15 will allow us to observe its downstream effects on the mediators of the JAK3/STAT3 pathway in the presence of cancer cell-secreted factors. Since our lab has previously shown IL-15 to promote a pro-oxidative muscle phenotype, and rescue fatigue in mouse models [3], we were interested in seeing its role in a cancer-induced model of muscle dysfunction. We first successfully established a working ex-vivo model using a $25 \%$ dilution of conditioned media 
from an established MDA-MB-231 breast cancer cell line, and then we examined the functional output of a whole muscle exposed to conditioned media. This gave us the ability to determine the relationship between muscle function and activation of the mediators of molecular pathways. This served as a translational tool since it simulated an in-vivo environment seen in tumorbearing animals through the models' common circulation between cancer cell-derived factors and muscle tissue. While this project only examined the JAK3/STAT3 pathway and used conditioned media from a specific breast cancer cell line, it was anticipated that this model can be used for examining additional signaling pathways using conditioned media from various cancer cell lines. For future work, therapeutic remedies and feasible dosing of IL-15 can also be tested since this model will serve as a translational tool as it can represent an in vivo scenario of shared circulation.

\section{CHAPTER 3.}

\section{Methods}

\subsection{Study Design Overview}

The project was completed in Dr. Emidio Pistilli's laboratory at West Virginia University. We used two different mice models - WT mice (strain C57BL/6J) and transgenic mice IL-15TG, which over express Interleukin-15 (measurable levels of IL-15 protein expression are approximately 6-fold greater in transgenic sera, in comparison to WT mice [67]). Each group was exposed to two different conditions: $25 \%$ diluted conditioned media (CM), and $25 \%$ diluted unconditioned media (UCM) prior to functional testing and molecular experiments. All of the 
conditioned and unconditioned media was collected from Dr. Elena Pugacheva's laboratory that is located at the West Virginia University Cancer Institute.

\subsection{Mice Models}

C57BL/6J WT mice were purchased from the Jackson Laboratory $(\mathrm{n}=18)$. The age matched WT mice were then randomly assigned to the two different groups: $25 \%$ unconditioned media (n=9), and 25\% conditioned media (n=9). $\boldsymbol{I L}-\mathbf{1 5 T G}$ mice were purchased from Jackson Laboratory $(n=20)$ and were bred to yield IL-15TG pups and littermate controls (no transgene present). The IL-15TG mice (both males and females) were then randomly assigned to four different groups: littermate control 25\% unconditioned media (TG -/- $n=5)$, transgenic 25\% unconditioned media ( $\mathrm{TG}+/-\mathrm{n}=5$ ), littermate control $25 \%$ conditioned media $(\mathrm{TG}-/-\mathrm{n}=5)$, and transgenic $25 \%$ conditioned media $(\mathrm{TG}+/-\mathrm{n}=5)$. Mice were housed in the AAALAC approved animal vivarium at West Virginia University at $22^{\circ} \mathrm{C}$ under a $12: 12$-h light-dark cycle and received food and water ab libitum. The Institutional Animal Care and Use Committee at West Virginia University approved all animal experiments prior to the start of the project.

\subsection{Animal Care}

All mice were housed in the animal vivarium located in the Robert C. Byrd Health Sciences Center (HSC) of West Virginia University School of Medicine. The animal vivarium maintains compliance with the NIH and the Institutional Animal Care and Use Committee guidelines. Animals were maintained in a pathogen-free environment, received adequate food and water and were attended to by a full-time veterinary doctor and support staff. 


\subsection{Media Collection}

The conditioned media was collected through the cultivation of the breast cancer cell line, MDA-MB-231. The cell culture procedures were performed in the Pugacheva lab under the same sterile conditions. The cells were maintained in high glucose DMEM supplemented with 10\% FBS to maintain active proliferation. Similar to the CM, the UCM comprised of DMEM and was plated at the same time and was exposed to identical conditions including incubation time, temperature and localization in the same incubator. The confluency of the cells was noted to ensure that there were no confounding errors. After the cells reached a confluency of greater than $90 \%$, the media was removed from the plate and was transported to the Pistilli lab where it was filtered using a 70micron filter and then placed into $1 \mathrm{~mL}$ tubes. The vials were immediately flash frozen in liquid nitrogen and stored in the -80 Celsius freezer until further use.

\subsection{Stock Preparation}

Prior to the surgical procedures, the two different chambers of the commercially available muscle physiology system (Aurora Scientific, Ontario, CA) were filled with the required level of media. The two tested conditions for the media were a) 25\% DMEM in Ringers Solution (forming our UCM) and b) 25\% Conditioned Media in Ringers Solution. DMEM is a medium for culturing different cell lines that contains amino acids, vitamins, and supplementary components (including Fetal bovine serum, growth factors, cytokines, matrix proteins, ATP, glucose, calcium etc.). Ringers Solution is made using $100 \mathrm{mM} \mathrm{NaCl}, 4.7 \mathrm{mM} \mathrm{KCl,} 3.4 \mathrm{mM}$ $\mathrm{CaCl} 2,1.2 \mathrm{mM} \mathrm{KH} 2 \mathrm{PO} 4,1.2 \mathrm{mM} \mathrm{MgSO} 4,25 \mathrm{mM}$ HEPES, and 5.5 mM D-glucose and distilled water. On the day of the experiment, the media was thawed in the water bath maintained at $37^{\circ} \mathrm{C}$ 
and mixed in with the Ringer solution that maintained a $\mathrm{pH}$ of 7.40 to reach a dilution of $25 \%$.

\subsection{Mouse Surgery}

On the day of data collection, each mouse was removed from the animal vivarium at West Virginia University and was brought to Pistilli Lab. Following the experimental set up, the mouse was placed under full anesthesia by breathing $4 \%$ isoflurane delivered through a nose cone at a flow rate of $1 \mathrm{~L} \cdot \mathrm{min}^{-1}$. The hind limbs of the mouse were taped down to expose the lateral side of the leg, and the Extensor Digitorum Longus (EDL) muscle was removed with both proximal and distal tendons intact (Figure 5). EDL muscles were immediately transferred to an oxygenated tissue bath that contained the Ringers solution maintained at $22^{\circ} \mathrm{C}$. While in the bath, nylon sutures were attached to the tendons. Dr. Emidio Pistilli performed all the surgical procedures, while I assisted during these procedures. Subsequently, the mouse was euthanized by cervical dislocation, while still under anesthesia. 


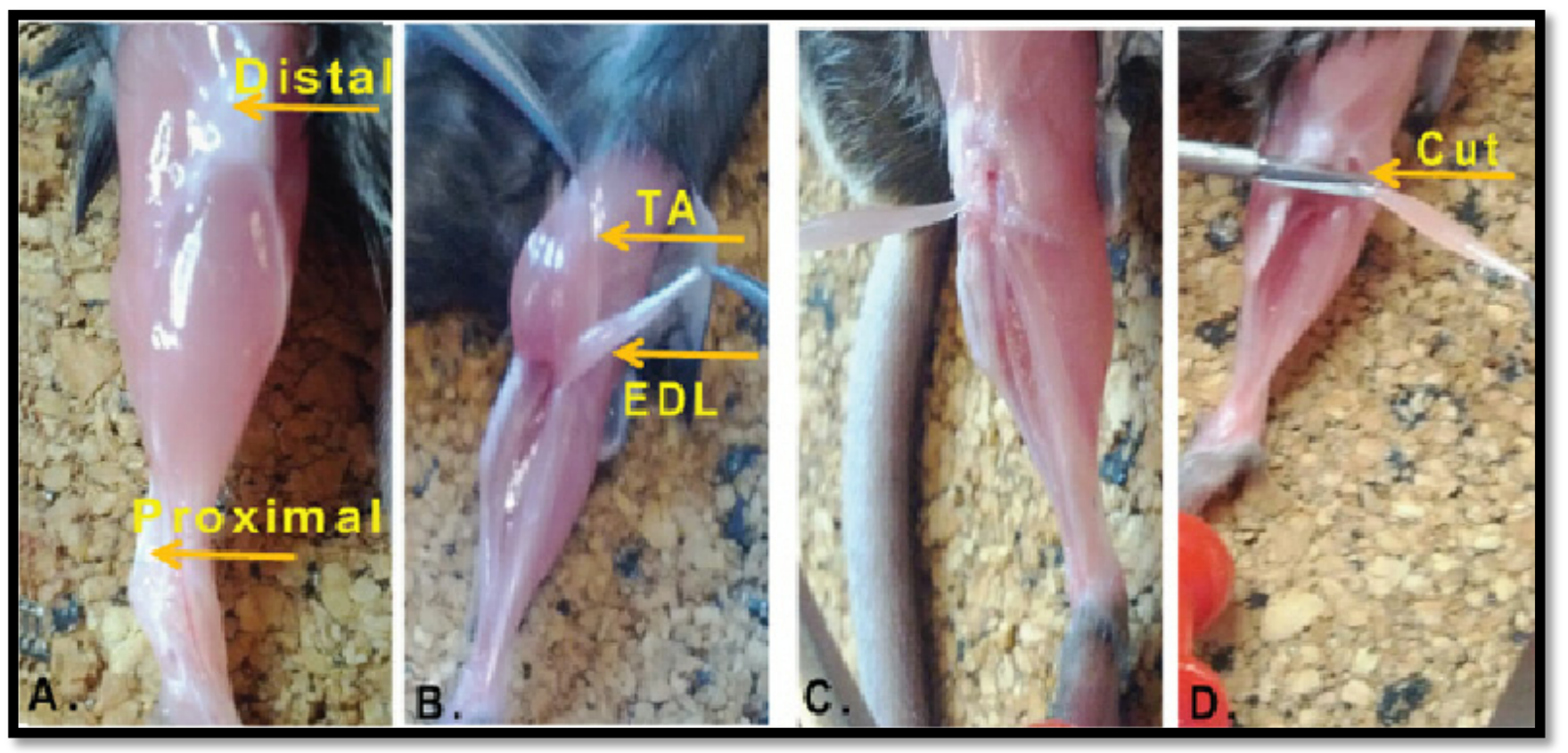

Figure 5: Extensor digitorum longus (EDL) muscle isolation.

This figure demonstrates the EDL muscle isolation from a mouse hindlimb. Panel $A$ shows the exposed hindlimb of an adult mouse with the arrows indicating the distal patella tendon and the proximal tendon attachments in the foot. Panel $\boldsymbol{B}$ shows the anatomical position of the Tibialis Anterior (TA) muscle and the EDL muscle. Panel C shows the EDL muscle being pulled superiorly to expose the distal tendon, while the proximal tendon is held in place with forceps. Panel D shows the tendon of the EDL being cut to release the EDL muscle [68].

\subsection{Muscle Function Analysis of EDL muscle in C57BL/6J WT mice, IL-15TG littermate control mice and IL-15TG overexpressing IL-15 mice.}

For the purpose of ex vivo muscle stimulation, a commercially available muscle physiology system (Aurora Scientific, Ontario, Canada) was used to assess the functional output of the EDL muscle in the different conditions. To remain consistent, the right EDL was set up in the chamber with the force transducer plate while the left EDL was set up in another incubation chamber in which the muscle was not stimulated (Figure 6). Both chambers were supplemented $95 \% / 5 \% \mathrm{O}_{2} / \mathrm{CO}_{2}$. For the EDL muscle that underwent contractions, a preset protocol that 
induced 3600 twitch contractions was selected. This protocol was repeated two consecutive times, to induce a total of 7200 twitch contractions. There was no resting period between the two consecutive protocol runs. The protocol duration was 3 hours and 10 minutes, which equated to 11,200 seconds. Therefore, we got a contraction every 1.6 seconds

(11200seconds/7200contractions). Once the protocol was completed, the muscles were removed from the chambers, the sutures were removed and the muscles were placed into labeled tubes. Immediately following that the vials were flash frozen in liquid nitrogen and stored in the -80 Celsius freezer until further use to assess molecular changes.

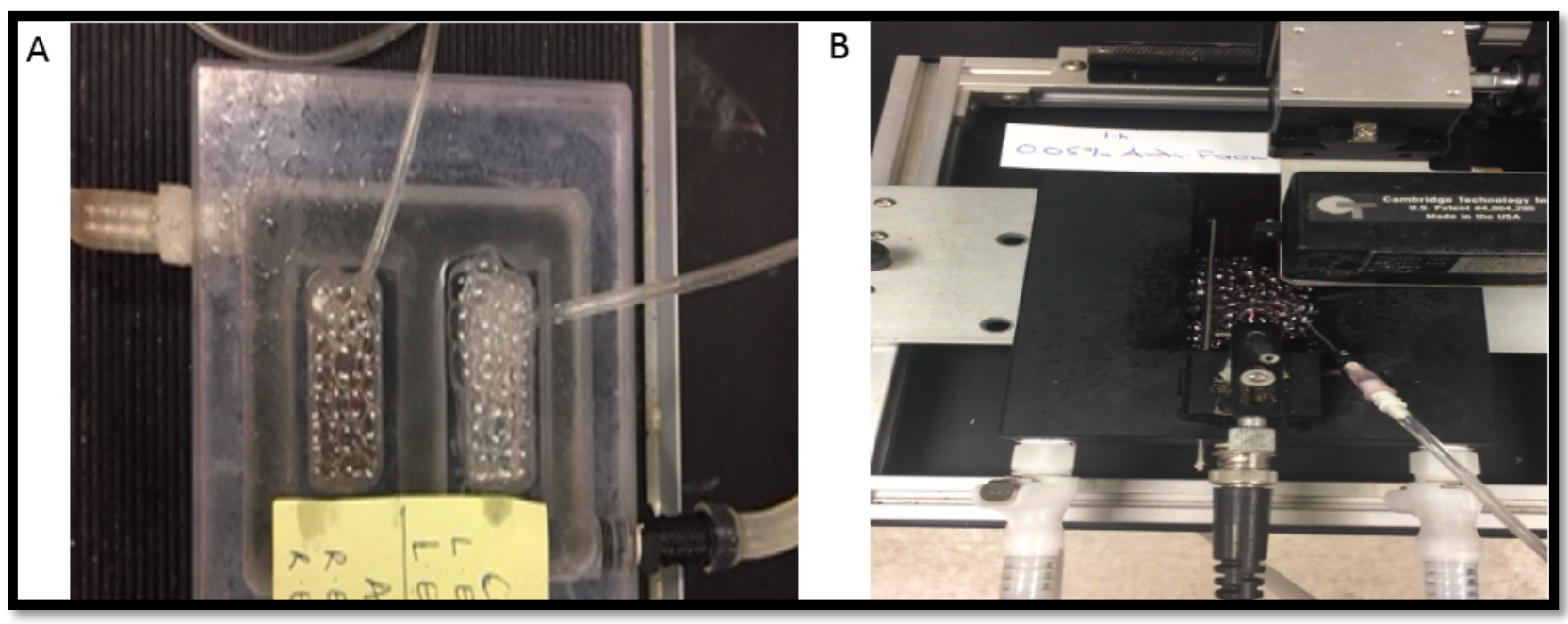

Figure 6: Aurora Scientific muscle physiology system.

This figure shows the physiology system - Aurora Scientific, Ontario, Canada, used to assess the functional output of the EDL muscle in the different conditions. Panel A shows the chamber in which the muscle exposed to media without any stimulations was placed in, whereas Panel B shows the chamber that was used to expose the muscle to media and used to test for function. 


\section{$3.8 \mathrm{pH}$ Measurement}

The $\mathrm{pH}$ of the specific media used was recorded at different time points during the protocol using the Thermo Scientific Orion Dual Star $\mathrm{pH}$ reader. The $\mathrm{pH}$ in the media was recorded at the start of the experiment for both the chambers, which was analogous. Once the 3 hour and 10 minute protocol was completed, the media was collected from the separate chambers and the $\mathrm{pH}$ was measured and recorded for both, the stimulated and unstimulated media.

\subsection{Protein Quantification}

To assess molecular changes through protein detection, the muscles that were previously flash frozen after functional testing were thawed and homogenized in preparation for western blot analyses. The entire EDL muscle was homogenized in RIPA buffer ( $1 \%$ sodium deoxycholate, $1 \%$ Triton $\mathrm{x}-100,150 \mathrm{mM} \mathrm{NaCl}, 5 \mathrm{mM}$ EDTA, $10 \mathrm{mM}$ Tris; $\mathrm{pH}$ 7.4), containing a phosphatase inhibitor and a protease inhibitor cocktail (Sigma-Aldrich, St. Louis, MO). Following homogenization, the protein concentrations were measured using the BioRad DC protein assay (BioRad, Hercules, CA).

\subsection{Western Blot}

To assess protein content, protein lysates were resolved using 4-12\% gradient polyacrylamide gels (PAGE) under denaturing conditions. Prior experience for successfully running PAGE gels had been achieved under the guidance of Dr. Yehenew Agazie at West Virginia University. Different key intermediates in the JAK3/STAT3 pathway were quantified. The protein concentrations were determined, and $20 \mu \mathrm{g}$ of protein was added to make a total of $30 \mu \mathrm{L}$ of loading samples, which were then loaded into each well of a 4-12\% gradient polyacrylamide gel (Invitrogen, Carlsbad, CA) and the gel was run at 120 
volts for 1 hour and 30 minutes. The proteins were transferred to a nitrocellulose membrane at $4^{\circ} \mathrm{C}$ for 1 hour at 25 volts in transfer buffer. Blocking non-specific protein binding was achieved by incubating the membranes in 5\% nonfat milk in Tris-buffered saline containing $0.05 \%$ Tween 20 (TBST) for 1 hour. The membranes were then incubated overnight with gentle rocking at $4{ }^{\circ} \mathrm{C}$ in appropriate primary antibodies of 1:1000 dilution. Following the overnight incubation, the membranes underwent $3 \times 10$ minute washes, followed by incubation in the appropriate secondary antibody (Invitrogen rabbit anti-mouse IgG-HRP or Invitrogen goat anti-rabbit IgG HRP, diluted 1:2000) at room temperature for 1 hour with gentle rocking. Membranes then underwent another 3×10 minute washes prior and the signals were developed by using enhanced chemiluminescence (Pierce ECL Western Blotting Substrate) that were visualized by a GE Amersham Imager 600, a high resolution imaging system to identify the band intensity. The digital images were quantified with Image $\mathbf{J}$ software (NIH) and the intensity of each band was normalized to GAPDH, which was from the same sample and on the same gel. Each membrane was stripped and reprobed between phospho-STAT3 probe and total-STAT3 probe. The data were expressed as arbitrary units.

\subsection{Protein Carbonyl Assay}

To assess if media induced oxidative stress induced any protein oxidation, the OxiSelect Protein Carbonyl Fluorometric Assay was used. The protein carbonyls in the different protein samples (1-10 mg/mL) were first derivatized with Protein Carbonyl Fluorophore (PCF). The PCF and protein carbonyl were bounded in a 1:1 ratio. Following this, the proteins were TCA precipitated and the free PCF was removed through acetone washes. The protein pellet was then dissolved in GuHCL, and the absorbance of the protein-fluorophore product was measured with a 485/538nm filter [69]. Lastly, the concentration of the protein carbonyl was calculated. 


\subsection{Data Analysis}

Statistical analyses were performed using GraphPad Prism 6 software. All data points collected from the twitch protocol were transported to excel prior to the software. Each individual group had a sample size of 5 muscles each $(n=5)$. Data are reported as mean \pm SEM, using two-way ANOVA statistical analyses. A p value of $\leq 0.05$ was considered to be statistically significant.

\section{CHAPTER 4. Results}

\subsection{Evaluation of Extensor Digitorum Longus (EDL) muscle function using different protocols.}

Preliminary studies to examine different protocols and their effect on inducing differences in mRNA levels of IL-6 were conducted in our lab. Table 1 shows the various conditions tested on extensor digitorum longus muscles (EDL) from C57BL/6J strain mice using a commercially available muscle physiology system (Aurora Scientific, Ontario, Canada). All functional procedures were performed in 100\% Ringers Solution $(100 \mathrm{mM} \mathrm{NaCl}, 4.7 \mathrm{mM} \mathrm{KCl}$, 3.4 mM CaCl2, 1.2 mM KH2PO4, 1.2 mM MgSO4, 25 mM HEPES, and 5.5 mM D-glucose, distilled water). Figure 7 and Figure 8 show the results of the semi-quantitative PCRs used to test the different conditions explained in Table 1. 
Table 1: Different Protocol Conditions.

The table below provides the specific details of the individual conditions used for analyzing stimulation of EDL muscles. Both left and right EDLs from the same mouse were tested with one serving as a control and the other being tested under the specific condition.

\begin{tabular}{|c|c|c|}
\hline \multicolumn{3}{|c|}{$\begin{array}{l}\text { Stimulation of EDL Muscles for Analysis under Different Protocol } \\
\text { Conditions }\end{array}$} \\
\hline \multirow[t]{2}{*}{ CONDITION 1} & \multirow[t]{2}{*}{ Control Normal Protocol } & EDL $1-$ Twitch $(1 \mathrm{~Hz})$, tetanus $(120 \mathrm{~Hz})$, fatigue \\
\hline & & $\begin{array}{l}\text { EDL } 2 \text { - Sit at Lo in oxygenated Ringer for } \\
\text { same time }\end{array}$ \\
\hline \multirow[t]{2}{*}{ CONDITION 2} & \multirow[t]{2}{*}{$\begin{array}{l}\text { Control Normal Protocol } \\
+30 \text { minute rest }\end{array}$} & $\begin{array}{l}\text { EDL } 1-\text { Twitch }(1 \mathrm{~Hz}) \text {, tetanus }(120 \mathrm{~Hz}) \text {, } \\
\text { fatigue, } 30 \text { minute rest }\end{array}$ \\
\hline & & $\begin{array}{l}\text { EDL } 2 \text { - Sit at Lo in oxygenated Ringer for } \\
\text { same time }\end{array}$ \\
\hline \multirow[t]{2}{*}{ CONDITION 3} & \multirow[t]{2}{*}{$\begin{array}{l}\text { Control Normal Protocol } \\
+60 \text { minute rest }\end{array}$} & $\begin{array}{l}\text { EDL } 1-\text { Twitch }(1 \mathrm{~Hz}) \text {, tetanus }(120 \mathrm{~Hz}) \text {, } \\
\text { fatigue, } 60 \text { minute rest }\end{array}$ \\
\hline & & $\begin{array}{l}\text { EDL } 2 \text { - Sit at Lo in oxygenated Ringer for } \\
\text { same time }\end{array}$ \\
\hline \multirow[t]{2}{*}{ CONDITION 4} & \multirow{2}{*}{$\begin{array}{l}\text { Twitch to get to Lo, } 1 \\
\text { twitch/ second for } 60 \\
\text { minutes, rest in bath for } \\
\text { same time (stimulate at } \\
1 \mathrm{~Hz} / 60 \mathrm{~min} \text { ) }\end{array}$} & $\begin{array}{l}\text { EDL } 1-1 \text { Twitch }(1 \mathrm{~Hz}) \text { per second for } 60 \\
\text { minutes, rest in bath for same time }\end{array}$ \\
\hline & & $\begin{array}{l}\text { EDL } 2 \text { - Sit at Lo in oxygenated Ringer for } \\
\text { same time }\end{array}$ \\
\hline \multirow[t]{2}{*}{ CONDITION 5} & \multirow{2}{*}{$\begin{array}{l}\text { Twitch to get to Lo, } 1 \\
\text { tetanus/ minute for } 60 \\
\text { minutes, rest in bath for } \\
\text { same time }\end{array}$} & $\begin{array}{l}\text { EDL } 1-1 \text { tetanus/ minute }(120 \mathrm{~Hz}) \text { for } 60 \\
\text { minutes, rest in bath for same time }\end{array}$ \\
\hline & & $\begin{array}{l}\text { EDL } 2 \text { - Sit at Lo in oxygenated Ringer for } \\
\text { same time }\end{array}$ \\
\hline \multirow[t]{2}{*}{ CONDITION 6} & \multirow{2}{*}{$\begin{array}{l}\text { Twitches to get to Lo, } \\
\text { then repeated twitches } \\
\text { every } 3 \text { seconds for } 3 \\
\text { hours + rest in bath for } 2 \\
\text { hours }\end{array}$} & $\begin{array}{l}\text { EDL } 1-\text { Twitches }(1 \mathrm{~Hz}) \text { every } 3 \text { seconds for } 3 \\
\text { hours or until }>50-70 \% \text { loss in force }+ \text { rest in } \\
\text { bath for } 2 \text { hours }\end{array}$ \\
\hline & & $\begin{array}{l}\text { EDL } 2 \text { - Sit at Lo in oxygenated Ringer for } \\
\text { same time }\end{array}$ \\
\hline \multirow[t]{2}{*}{ CONDITION 7} & \multirow{2}{*}{$\begin{array}{l}\text { Twitches to get to Lo, } \\
\text { then repeated tetanus } \\
\text { once per minute for } 3 \\
\text { hours + rest in bath for } 2\end{array}$} & $\begin{array}{l}\text { EDL } 1 \text { - repeated tetanus }(120 \mathrm{~Hz}) \text { once per } \\
\text { minute for } 3 \text { hours or until }>70 \% \text { loss in force }+ \\
\text { rest in bath for } 2 \text { hours }\end{array}$ \\
\hline & & EDL 2 - Sit at Lo in oxygenated Ringer for \\
\hline
\end{tabular}




\begin{tabular}{|c|c|c|}
\hline & hours & same time \\
\hline \multirow[t]{2}{*}{ CONDITION 8} & \multirow[t]{2}{*}{$\begin{array}{l}\text { Incubation with elevated } \\
\text { temperature }\left(37^{\circ} \mathrm{C}\right) \text { for } 2 \\
\text { hours }\end{array}$} & $\begin{array}{l}\text { EDL } 1 \text { - Incubation with elevated temperature } \\
\left(37^{\circ} \mathrm{C}\right) \text { for } 2 \text { hours }\end{array}$ \\
\hline & & $\begin{array}{l}\text { EDL } 2 \text { - Incubation with normal temperature } \\
\left(22^{\circ} \mathrm{C}\right) \text { for } 2 \text { hours }\end{array}$ \\
\hline \multirow[t]{2}{*}{ CONDITION 9} & \multirow{2}{*}{$\begin{array}{l}\text { Incubation with } \\
\text { depressed temperature } \\
\left(9-10^{\circ} \mathrm{C}\right) \text { for } 2 \text { hours }\end{array}$} & $\begin{array}{l}\text { EDL } 1-\text { Incubation with depressed temperature } \\
\left(9-10^{\circ} \mathrm{C}\right) \text { for } 2 \text { hours }\end{array}$ \\
\hline & & $\begin{array}{l}\text { EDL } 2 \text { - Incubation with normal temperature } \\
\left(22^{\circ} \mathrm{C}\right) \text { for } 2 \text { hours }\end{array}$ \\
\hline
\end{tabular}

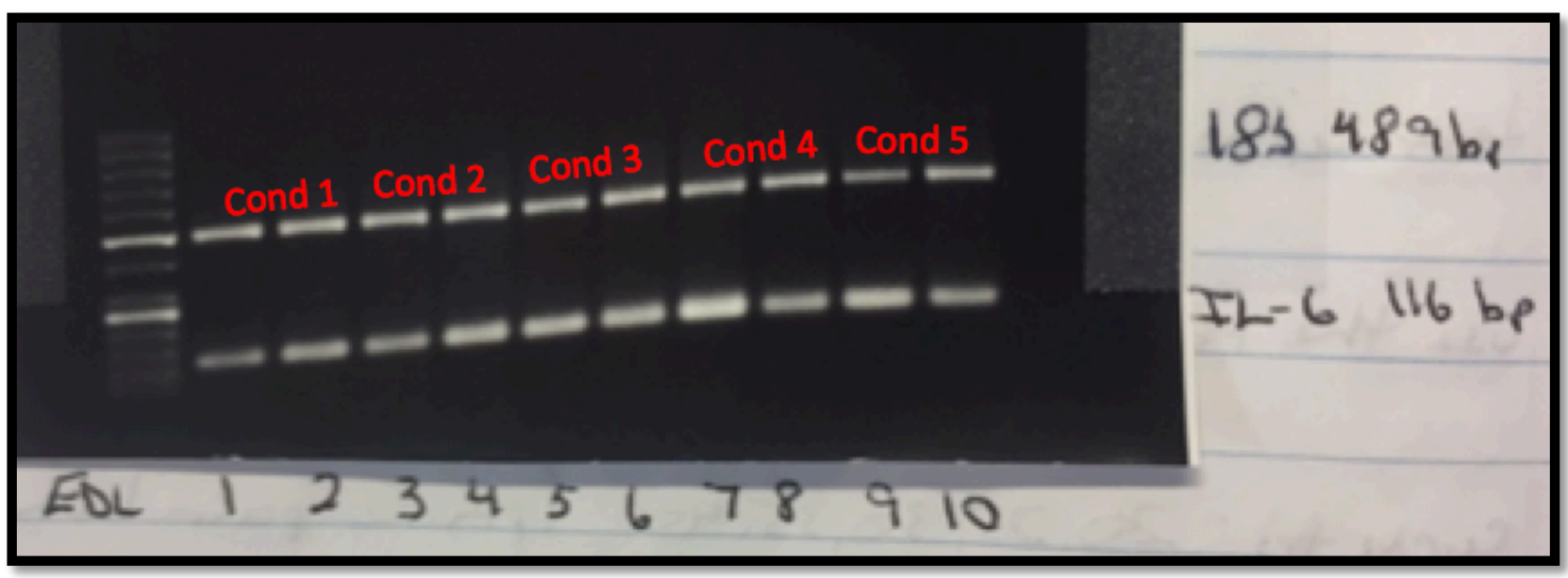

Figure 7: PCR depicting mRNA levels of IL-6.

This semi-quantitative PCR shows the mRNA levels of IL-6 obtained from the EDL muscles of muscles placed in the following conditions: condition 1 (EDL 1 and EDL 2), condition 2 (EDL 3 and EDL 4), condition 3 (EDL 5 and EDL 6), condition 4 (EDL 7 and EDL 8), and condition 5 (EDL 9 and EDL 10). Increases in $m R N A$ levels were observed in EDL muscles 7 and 9 corresponding to condition 4 (twitch $(1 \mathrm{~Hz})$ allowed to get to Lo, 1 tetanus $(120 \mathrm{~Hz}) /$ minute for 60 minutes) and condition 5 (twitch (1Hz) to get to Lo, 1 tetanus $(120 \mathrm{~Hz}) /$ minute for 60 minutes), respectively. 


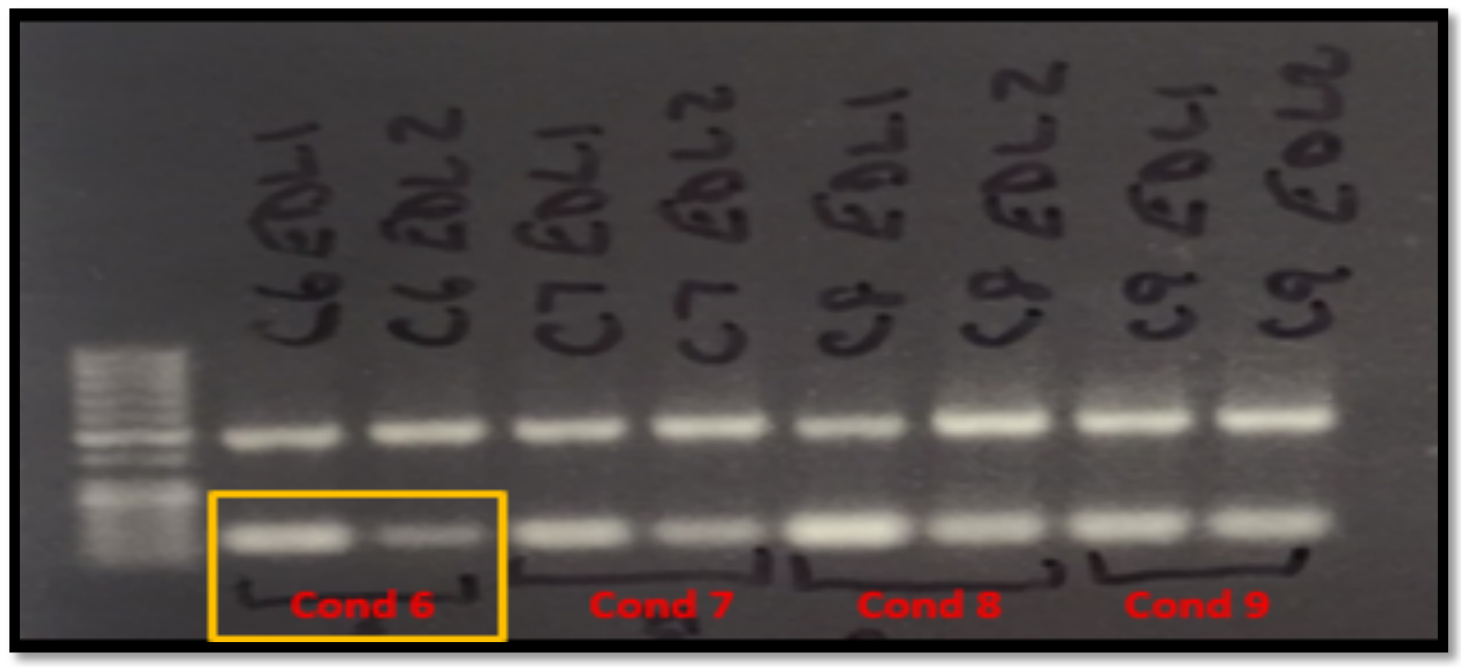

Figure 8: PCR depicting mRNA levels of IL-6.

This semi-quantitative PCR shows the mRNA levels of IL-6 obtained from the EDL muscles placed in the following conditions: condition 6 (EDL 1 and EDL 2), condition 7 (EDL 3 and EDL 4), condition 8 (EDL 5 and EDL 6), and condition 9 (EDL 7 and EDL 8). Increases in $m R N A$ levels were observed in EDL muscles 1 and 2 corresponding to condition 6 (Twitches allowed to get to maximal twitch response (i.e., Lo), then repeated twitches $(1 \mathrm{~Hz})$ every 3 seconds for 3 hours or until $>50-70 \%$ loss in force + rest in bath for 2 hours).

We found that 3 hours of twitches $(1 \mathrm{~Hz})$ produced a larger IL-6 response than the 3-hour tetanus $(120 \mathrm{~Hz})$ (Figure 8). These data suggested that maximal change in the mRNA levels was observed when the protocol is run under condition 6 (Twitches allowed to get to maximal twitch response (i.e., Lo), then repeated twitches $(1 \mathrm{~Hz})$ every 3 seconds for 3 hours or until $>50-70 \%$ loss in force + rest in bath for 2 hours). Therefore, we decided to address Specific Aim \#1 of the project, which is to determine the effects of $25 \%$ diluted conditioned media on muscle function in an ex vivo model, using condition 6 for our experimental protocol.

\subsection{Determination of Conditioned Media Composition for Muscle Incubation}

To determine what percentage of conditioned media (CM) from the culture MDA-MB-

231 cell line will be appropriate for the purpose of the model, semi-quantitative PCR for Muscle atrophy F-box (MAFbx)/Atrogin-1, Muscle RING finger 1 (Murf-1), and Interleukin (IL-6) were 
performed. Murf-1 and Atrogin-1 are two muscle-specific E3 ubiquitin ligases that have shown to be transcriptionally increased in skeletal muscles under atrophy-inducing conditions [70]. On the other hand, IL-6 is suggested as a potential mediator in cancer cachexia, which is seen in the acute phase of inflammation associated in cancer cachexia [71]. Consistent with previously established work, we observed an increase in mRNA levels in both, Murf-1 and Atrogin-1, markers of muscle atrophy using $25 \%$ diluted CM in comparison to the samples exposed to either $10 \%$ diluted $\mathrm{CM}$ or control media (Figure 9).

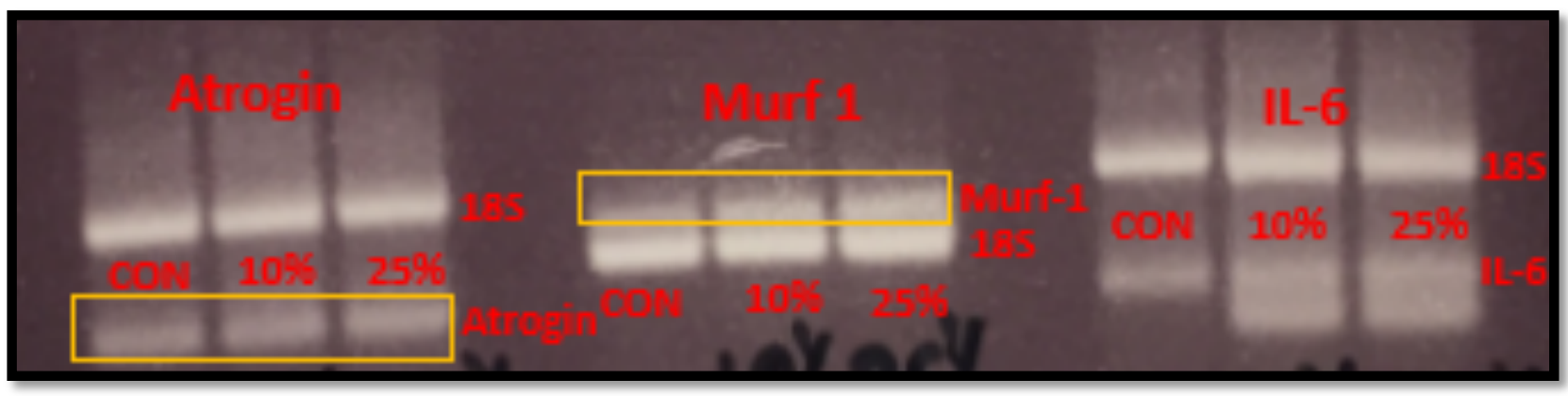

Figure 9: PCR depicting mRNA levels of Murf-1, Atrogin-1, and IL-6 using different CM percentages. This semi-quantitative PCR shows the mRNA levels of three biomarkers; Atrogin1, Murf1, and IL-6 in EDL muscles exposed to control media, EDL muscles exposed to $10 \%$ diluted CM, and EDL muscles exposed to 25\% diluted CM. Changes were observed in Atrogin1 and Murf1 in the samples exposed to 25\% diluted CM in comparison to the samples exposed to either $10 \%$ diluted CM or control media.

\subsection{Effect of 25\% diluted CM on Contractile Data of muscles exposed to secreted factors from the MDA-MB-231 cell line}

To test the muscle function in this cancer-induced model of muscle dysfunction, we measured muscle force in different mice models - C57BL/6J WT mice, IL-15TG, overexpressing IL-15, and IL-15TG littermate controls. Figures $10-13$ are representative of the results from the 
experimental protocol. Muscles were stimulated using 1 twitch every 1.6 seconds for 3 hours and 10 minutes giving us a total of 7200 contractions. The EDL Fatigue Curves represent the relative percentage of fatigue seen across all groups and media exposure. We then calculated the total aggregate force of the entire protocol time to get the Area Under the Curve (AUC) graphs, which can be equated as the total force output during the protocol.

Figure 10 represents the EDL Fatigue curve and AUC for the WT and TG littermate control groups exposed to either CM or UCM. This validated our assumption of seeing no statistical difference between these two groups. With the results of this control experiment, we decided to analyze TG (IL-15TG) and TG controls for further muscle contractile analysis. A combined analysis of all three groups (WT, TG, and TG controls) is reported in the supplementary data as Supplementary Figure 1 and Supplementary Figure 2, respectively.

Figure 11 represents the EDL Fatigue curve and AUC for the TG and TG Control groups exposed to the two media types (CM vs. UCM). No statistical differences are seen in either of the groups. We were also interested in comparing all three genotypic groups relative to either $\mathrm{CM}$ or UCM to see any statistical significance or trend. Figure 12 and Figure 13 report these analyses. No statistical significance were seen in either of the analyses. We concluded that acute exposure to conditioned media produces no significant effect on muscle fatigue. 


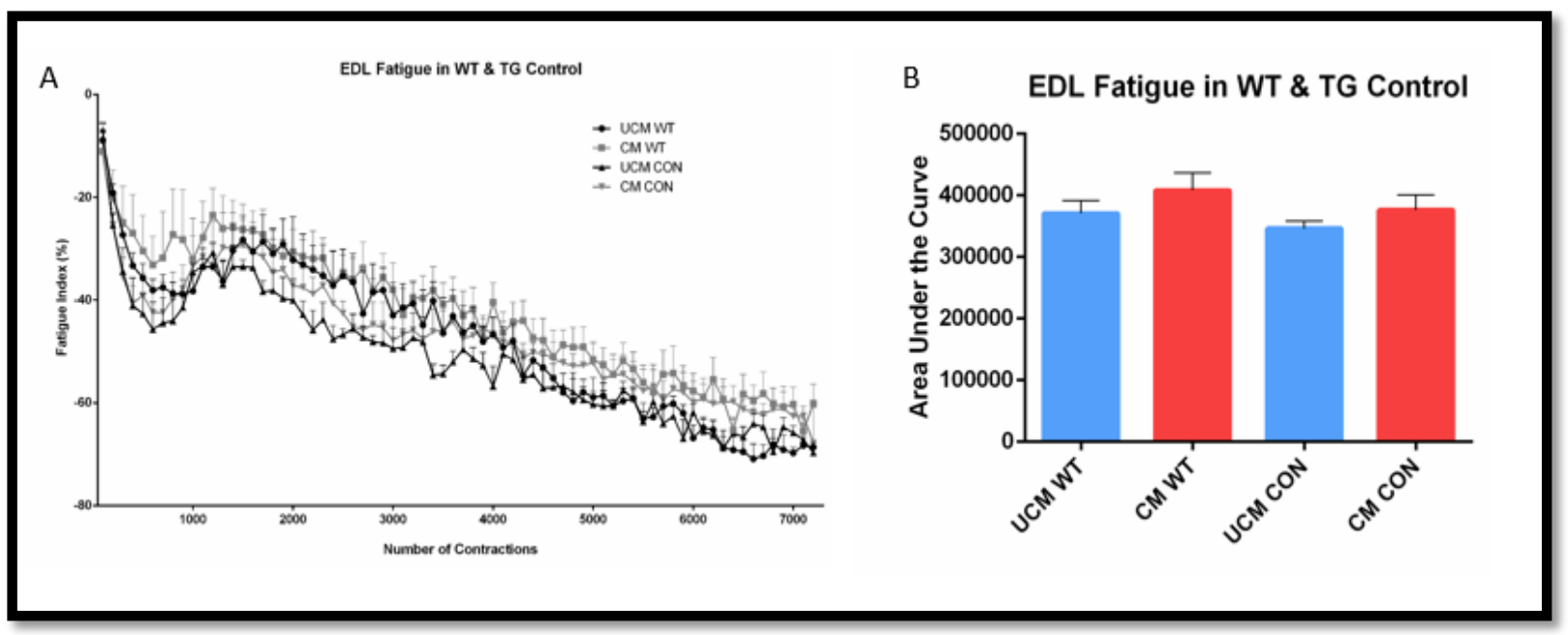

Figure 10: EDL Fatigue curve and Area Under the Fatigue Curve for WT and TG Control. Panel $\boldsymbol{A}$ shows the EDL fatigue curves for conditions in the UCM WT, CM WT, UCM CON, and CM CON groups. No statistical differences were seen between any of the groups. Panel B shows the EDL Total Area Under the Fatigue Curves $\left(m N^{*} S\right)$ for conditions in the UCM WT, CMWT, UCM CON, and CM CON groups. No statistical differences were seen between any of the group.

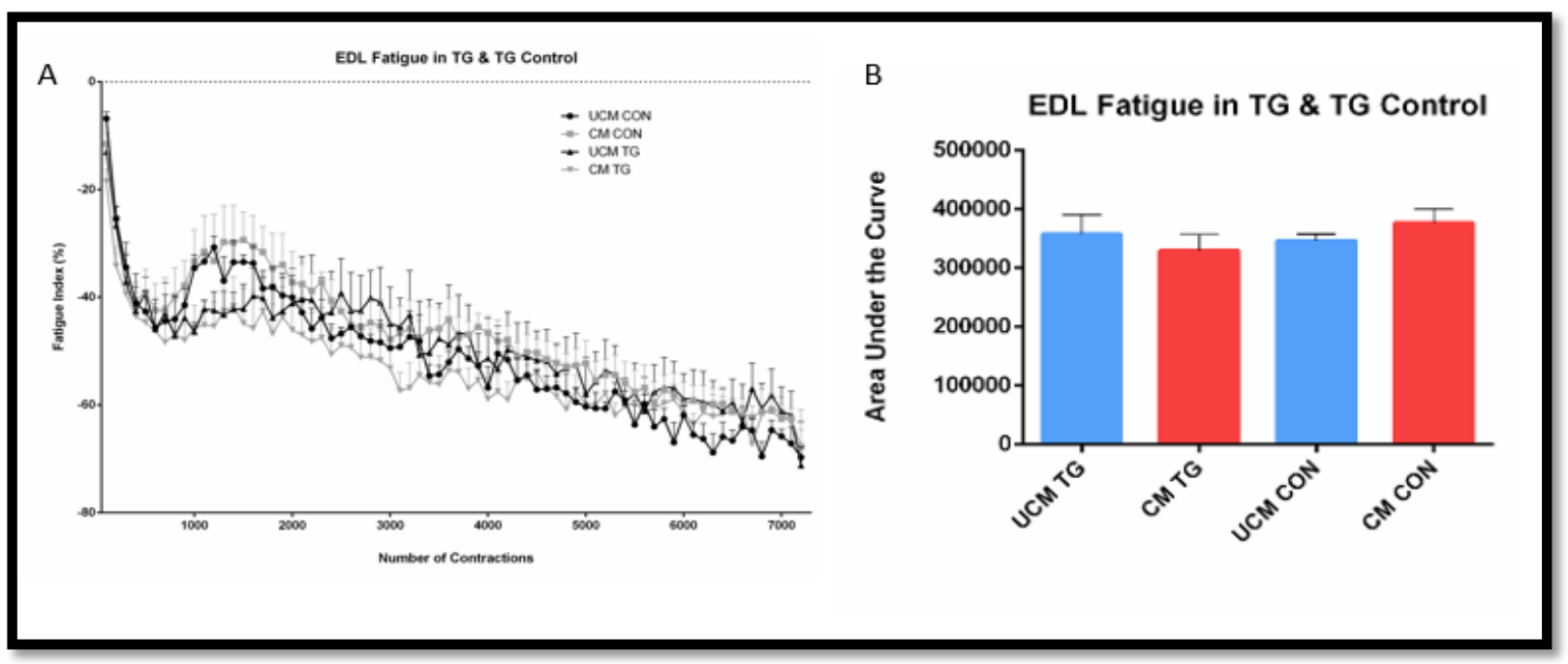

Figure 11: EDL Fatigue curve and Area Under the Fatigue Curve for TG and TG Control. Panel $\boldsymbol{A}$ shows the EDL fatigue curves for conditions in the UCM TG, CM TG, UCM CON, and CM CON groups. No statistical differences were seen between any of the groups. Panel B shows the EDL Total Area Under the Fatigue Curves ( $\left.m N^{*} S\right)$ for conditions in the UCM TG, CM TG, UCM CON, and CM CON groups. No statistical differences were seen between any of the group. 


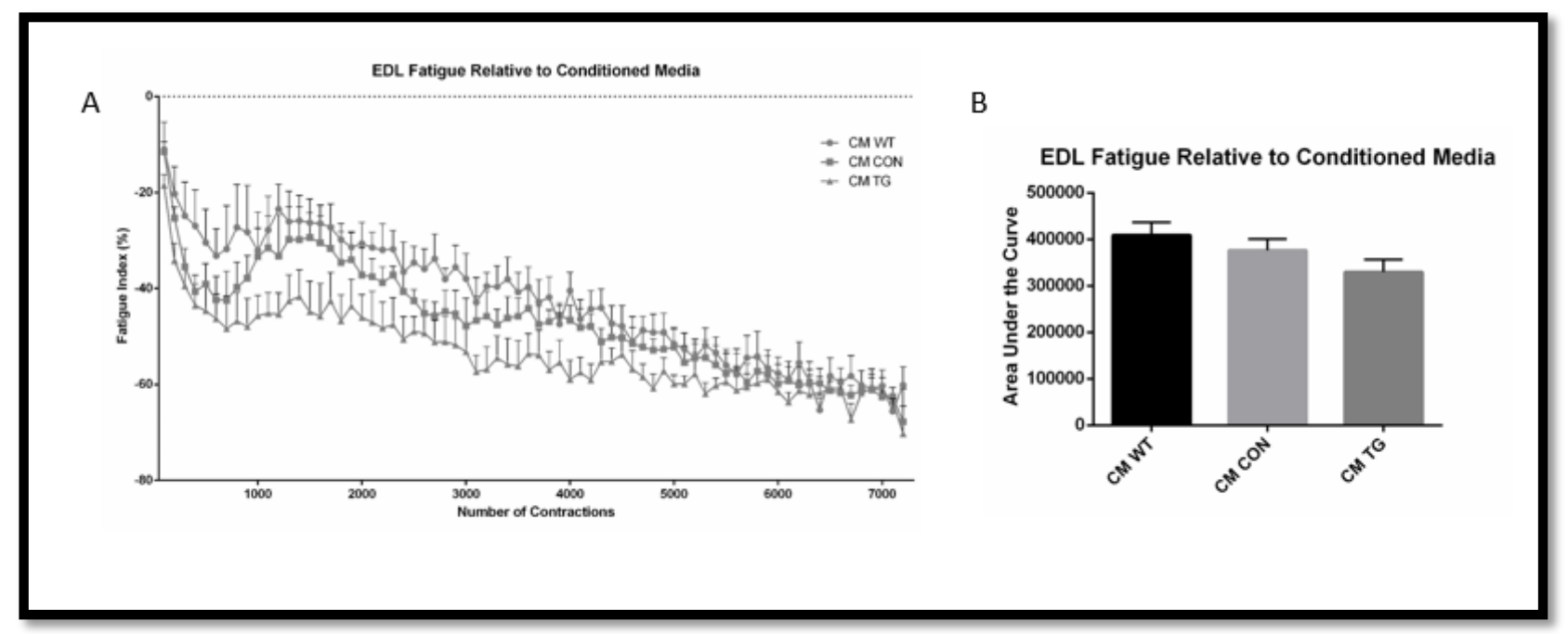

Figure 12: EDL Fatigue curve and Area Under the Fatigue Curve for all groups exposed to $25 \%$ diluted CM

Panel $\boldsymbol{A}$ shows the EDL fatigue curves for all three groups (WT, TG, and CON) exposed to 25\% diluted CM. No statistical differences were seen between any of the groups. Panel B shows the EDL Total Area Under the Fatigue Curves $\left(m N^{*} S\right)$ for all three groups (WT, TG, and CON) exposed to 25\% diluted CM. No statistical differences were seen between any of the groups.

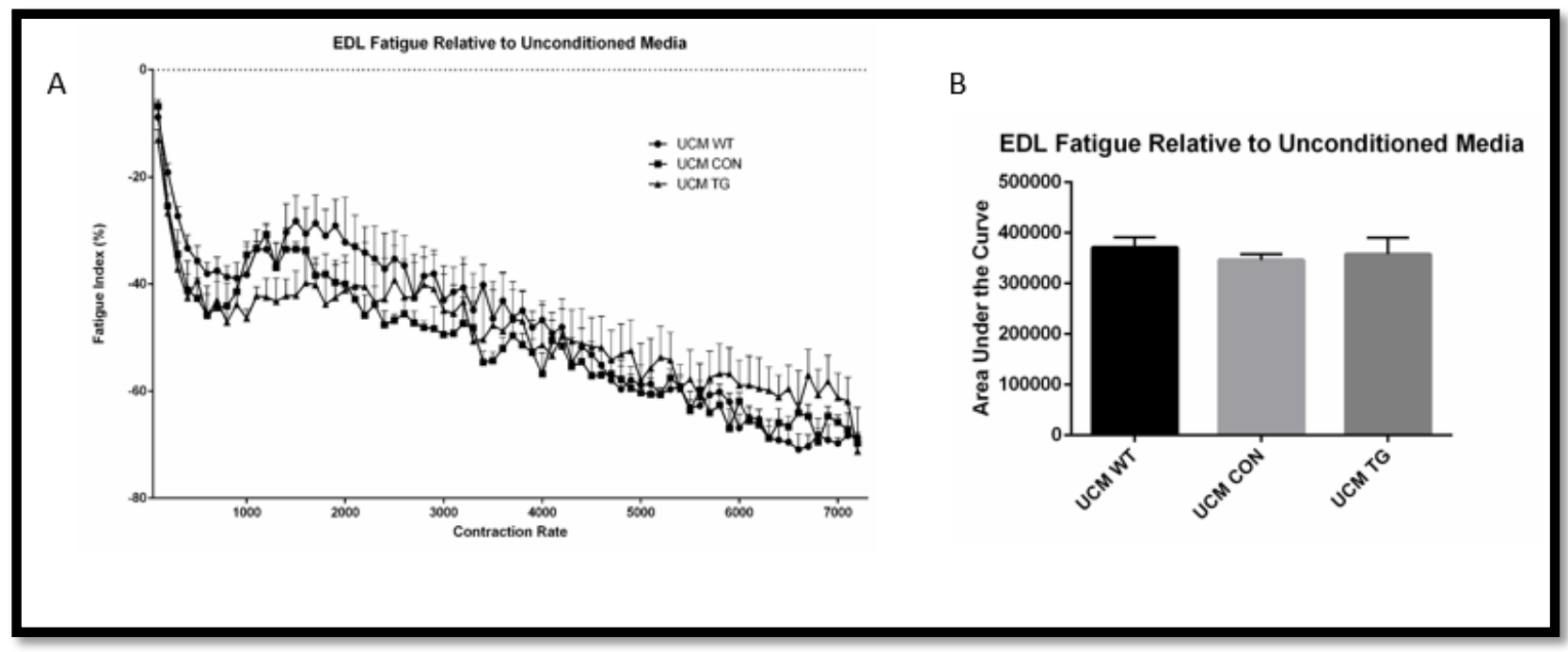

Figure 13: EDL Fatigue curve and Area Under the Fatigue Curve for all groups exposed to 25\% UCM

Panel $\boldsymbol{A}$ shows the EDL fatigue curves for all three groups (WT, TG, and CON) exposed to 25\% diluted UCM. No statistical differences were seen between any of the groups. Panel $\boldsymbol{B}$ shows the EDL Total Area Under the Fatigue Curves ( $\left.m N^{*} S\right)$ for all three groups (WT, TG, and CON) exposed to 25\% diluted UCM. No statistical differences were seen between any of the groups. 


\subsection{Protein Carbonyl Concentrations to measure for Oxidative Stress}

To assess if oxidative stress of media treatments induced muscle protein oxidation, the OxiSelect Protein Carbonyl Fluorometric Assay was used. We decided to combine all the genotypic samples together to analyze for carbonyl concentrations relative to either stimulation (stimulated vs. unstimulated) and media type (CM vs. UCM). Figure 14 is represntative of these analyses. No statistical differences were seen between any of the groups. We concluded that acute exposure to conditioned media cell-secreted factors, which we hypothesized would lead to oxidative stress did not induce any significant protein carbonylation between the different groups.

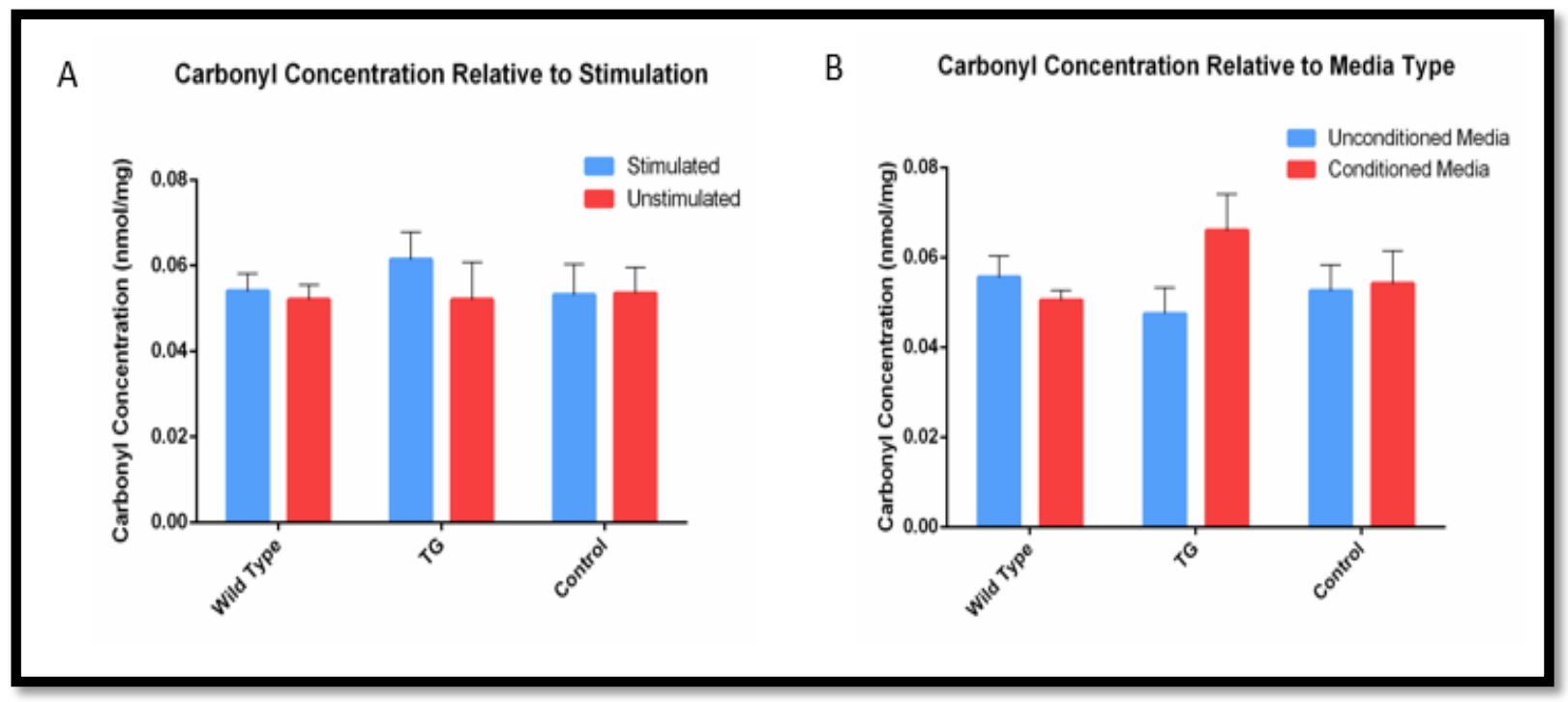

Figure 14: Carbonyl Concentration (nmol/mg) in all groups relative to different conditions. Panel $\boldsymbol{A}$ shows the Carbonyl concentrations present in the different groups (WT, TG, and CON) relative to muscle stimulation (stimulated vs. unstimulated). No statistical differences were seen between any of the groups. Panel $\boldsymbol{B}$ shows the Carbonyl concentrations present in the different groups (WT, TG, and CON) relative to the media type (CM vs. UCM). No statistical differences were seen between any of the groups. 


\subsection{Measurement of $\mathrm{pH}$ changes}

Another factor implicated for the development of muscle fatigue is the presence of excess protons $\left(\mathrm{H}^{+}\right)$that arise as a by-product of glycolysis $[70,71]$. To assess if the $\mathrm{pH}$ change was significantly different between the media type and stimulations, we noted the $\mathrm{pH}$ of the media prior to the start of the protocol and at the end of the protocol in each condition for the samples in the Wild Type group. The percentage change was then put into our statistical model and Figure 14 is represntative of these analyses. No statistical differences were seen between any of the groups. Since contractile activity has been shown to lead to an acidotic state that has further been related to muscle fatigue $[72,73]$, we concluded that our sample size was too small to show significance as we only measured $\mathrm{pH}$ changes in the WT group $(\mathrm{n}=8)$.

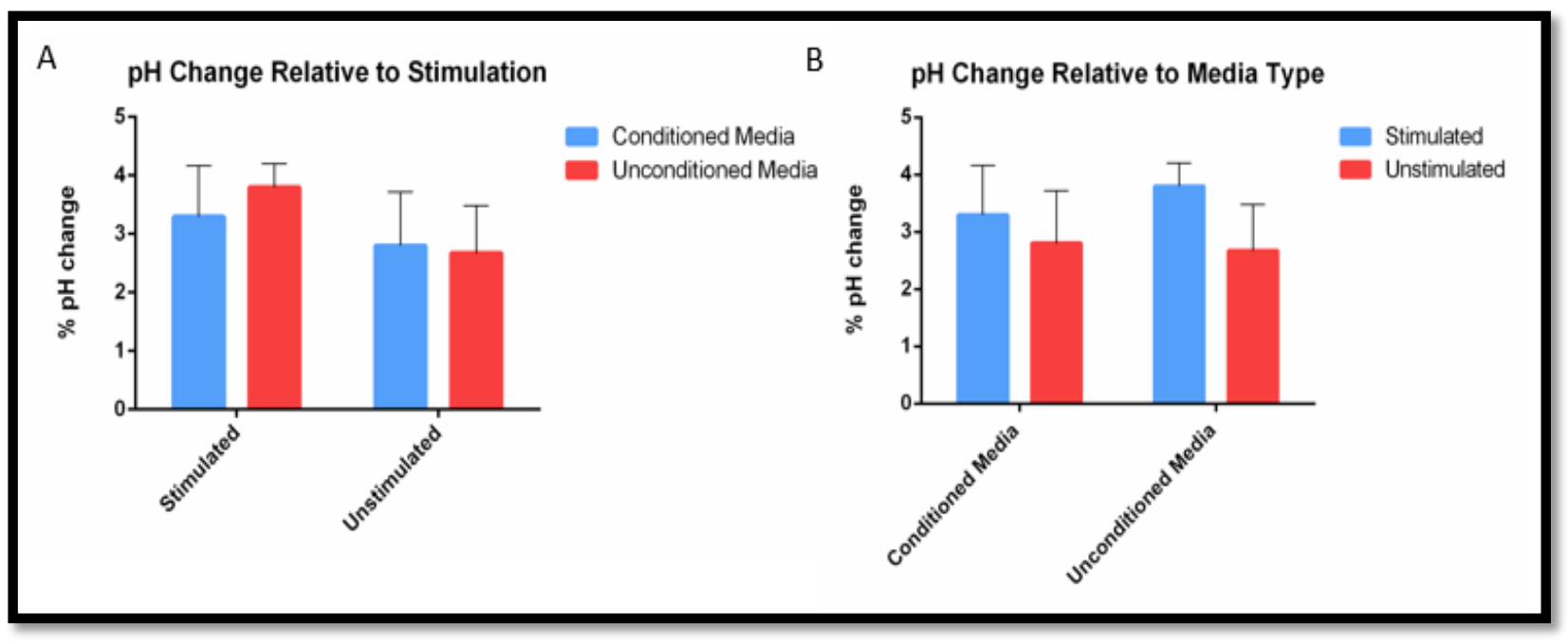

Figure 15: Measurement of $\mathbf{p H}$ changes in the Wild Type group.

Figure 15 shows the $\mathrm{pH}$ changes that were measured at two different time points - prior to the start of the experiment protocol and at the end of the protocol in the Wild Type group. Panel $\boldsymbol{A}$ shows the grouped $\mathrm{pH}$ change comparing media type (CM vs. UCM), while Panel B shows the grouped pH change comparing muscle stimulation (stimulated vs. unstimulated). No statistical differences were seen between any of the groups. 


\subsection{Effect of 25\% diluted CM on Contractile Data of muscles following 2-hour Extended Exposure}

Towards the end of the project, with only two mice left in both our TG and TG Control colonies, we decided to alter our protocol to extend the exposure time of the MDA-MB-231 cellsecreted factors on the muscles. Recent studies have reported an effect on signaling pathways by CM only after a chronic exposure $[9,14]$. Based on this evidence, we decided to assign each of the 4 mice to the 4 different groups (CM TG, UCM TG, CM CON, and UCM CON). We used the same functional output protocol, but added an additional 2-hour pre-incubation in the respective media and chamber. A continuous line of $100 \%$ oxygen was supplied to each muscle. Since we only had one sample in each group, no statistical analyses were performed. However, a graphical comparison and representation between the mean of the fatigue curves of the respective groups with the muscle fatigue curve of the individual samples with Extended Exposure are presented in Figures 16 and 17. The blue fatigue curves represented in Panel A and C of Figure 16 are indicative of the mean fatigue curves of the TG mice and TG Control mice exposed to UCM in the experiments that were already conducted without extended exposure, while the orange fatigue curves are indicative of the fatigue curves of the single muscles that were pre-incubated for an additional two hours prior to the start of the fatigue protocol. Similarly, the blue fatigue curves in Panel A and C of Figure 17 are indicative of the mean fatigue curves of the TG mice and TG Control mice exposed to CM in the experiments that were already conducted without extended exposure, while the orange fatigue curves are indicative of the fatigue curve of the single muscles that were pre-incubated for an additional two hours prior to the start of the fatigue protocol. We observe a shift in the EDL fatigue curve of the single 
muscles (orange fatigue curve) to the bottom left in all 4 samples relative to their groups. This suggests that the additional pre-incubation may in fact be causing muscle dysfunction via exposure top cancer cell-secreted factors. Similarly, we observed a decrease in Area Under the Fatigue Curve for all of the samples, coinciding with the graphs, thereby showing a lower total aggregate force.

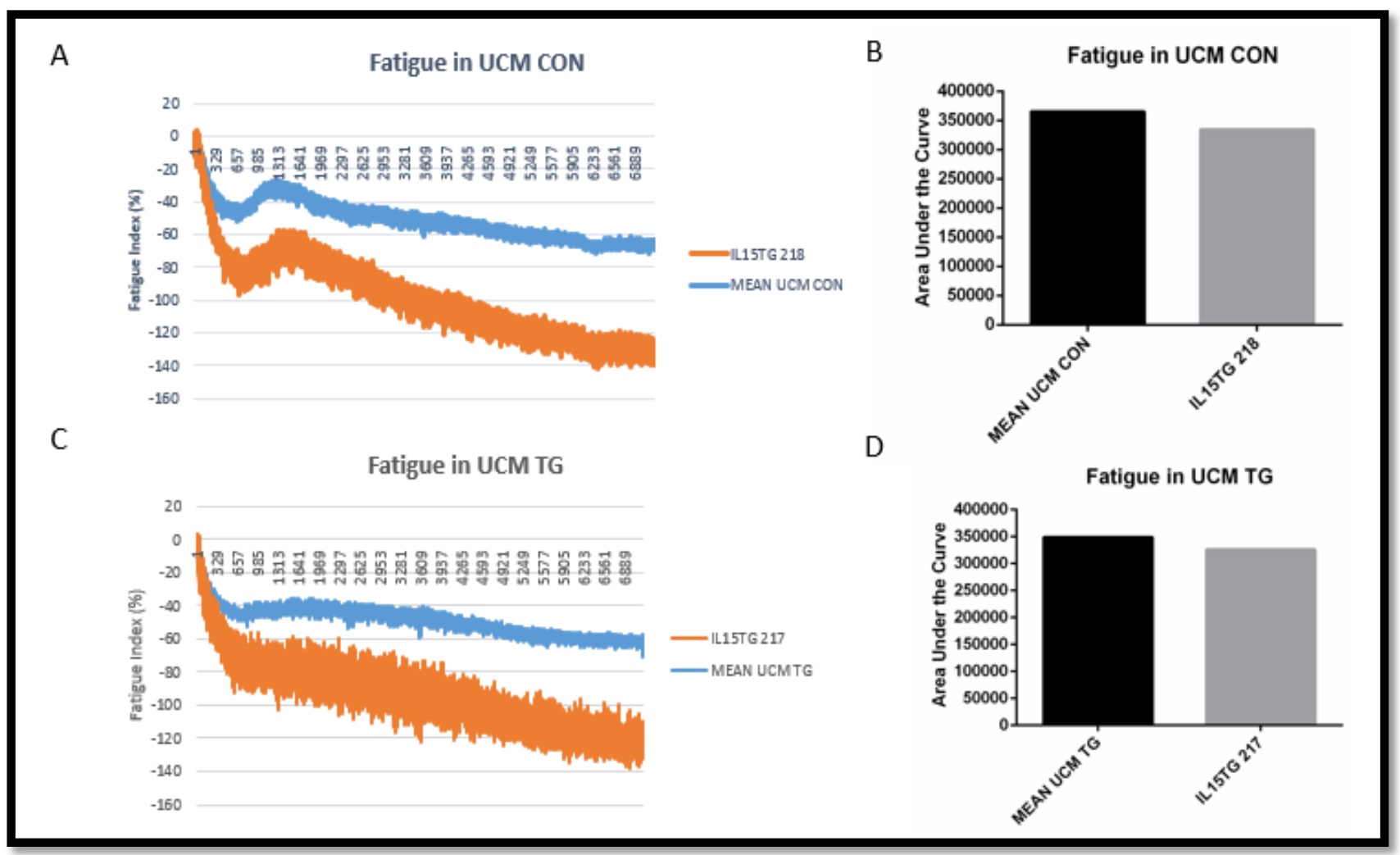

Figure 16: EDL Fatigue curves and Area Under the Fatigue Curve for UCM CON and UCM TG groups exposed to their respective Extended Exposure Sample

Panel $\boldsymbol{A}$ shows the EDL fatigue curves for the mean UCM CON group and the Extended Exposure sample. Panel B shows the EDL Total Area Under the Fatigue Curves for the mean UCM CON group and the Extended Exposure sample. Panel C shows the EDL fatigue curves for the mean UCM TG group and the Extended Exposure sample. Panel D shows the EDL Total Area Under the Fatigue Curves for the mean UCM TG group and the Extended Exposure sample. 


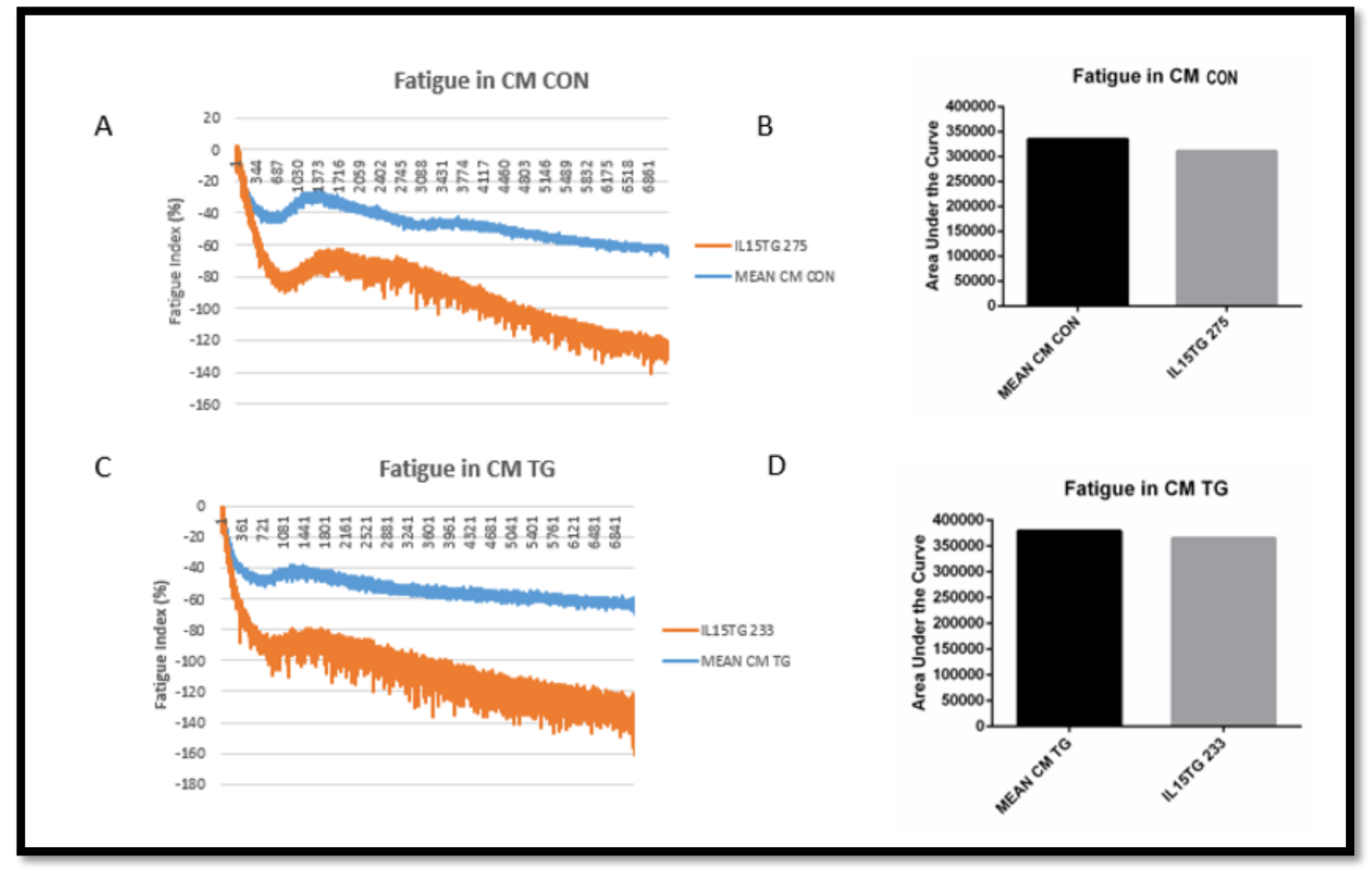

Figure 17: EDL Fatigue curves and Area Under the Fatigue Curve for CM CON and CM TG groups exposed to their respective Extended Exposure Sample

Panel $\boldsymbol{A}$ shows the EDL fatigue curves for the mean CM CON group and the Extended Exposure sample. Panel B shows the EDL Total Area Under the Fatigue Curves $\left(m N^{*} S\right)$ for the mean CM $C O N$ group and the Extended Exposure sample. Panel $C$ shows the EDL fatigue curves for the mean CM TG group and the Extended Exposure sample. Panel D shows the EDL Total Area Under the Fatigue Curves ( $\left.m N^{*} S\right)$ for the mean CM TG group and the Extended Exposure sample.

\subsection{Western Blot Analysis of pSTAT3 and total STAT3}

Western blot analyses were performed on WT and TG samples exposed to the different conditions (CM stimulated, CM unstimulated, UCM stimulated, and UCM unstimulated). For protein quantification, all of the signals from the proteins of interest (pSTAT3 and STAT3) were first normalized to GAPDH, which was probed on the same gel. Figure 18 represents the western blots for all of the samples in the WT group. No significance was observed in either of the samples (WT group, $\mathrm{n}=16)$. However, a statistical significance $(\mathrm{p}<0.05)$ was seen in 
phosphorylated STAT3 to total STAT3 in the stimulated CM and the stimulated UCM conditions in the TG samples (TG group, $n=16$ ). Since the samples in both groups were combined, we ran additional analyses on the individual gels $(\mathrm{n}=8)$, which are represented as Supplementary Figures 4 through 8 . No significant differences were seen in either of the individual analyses in both WT samples and TG samples.

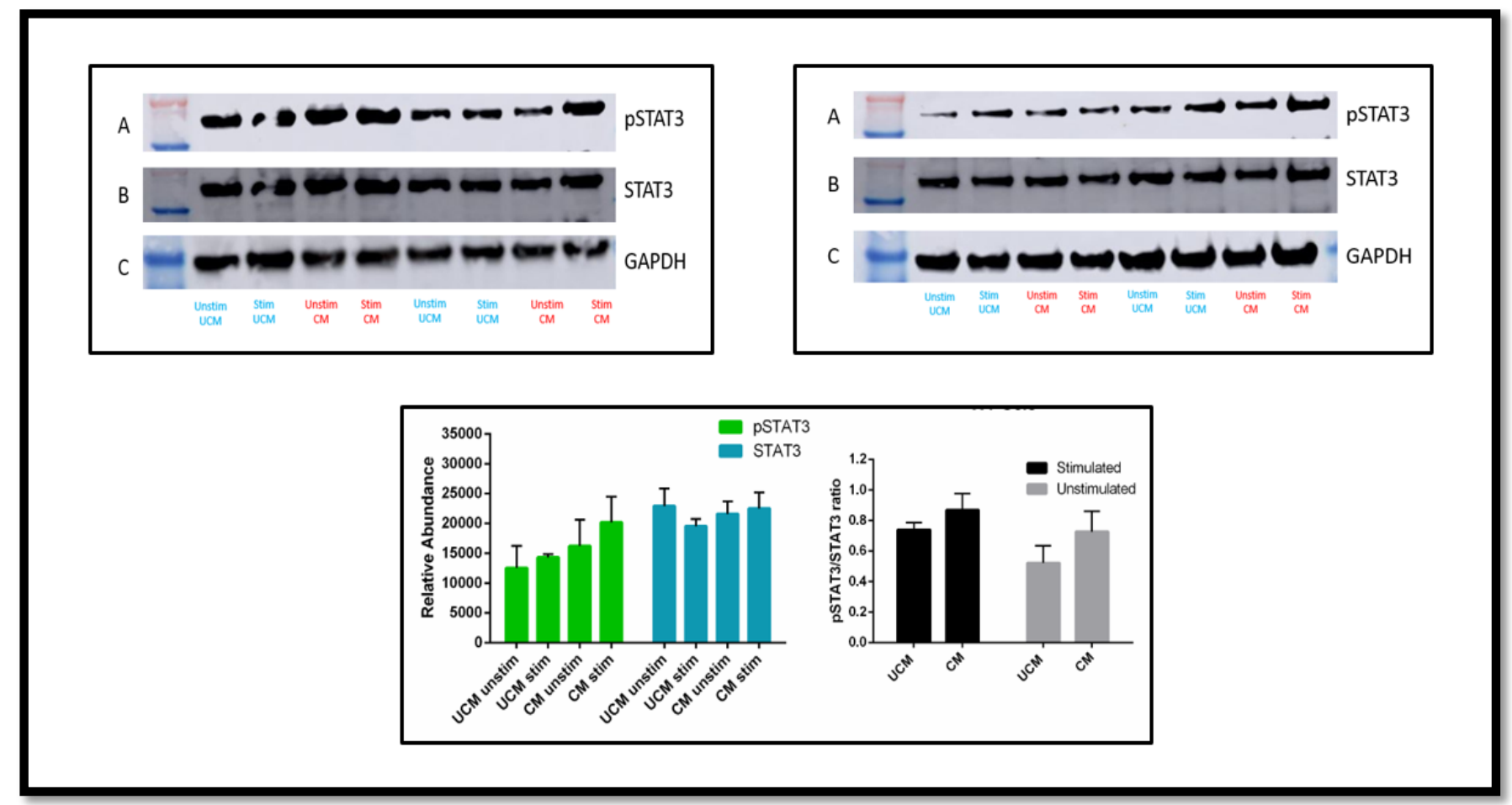

Figure 18: Quantification of pSTAT3 and STAT3 in combined analysis $(n=16)$ for the WT samples.

Quantification of the WT samples showed no statistical significance in phosphorylated STAT3 to total STAT3. 


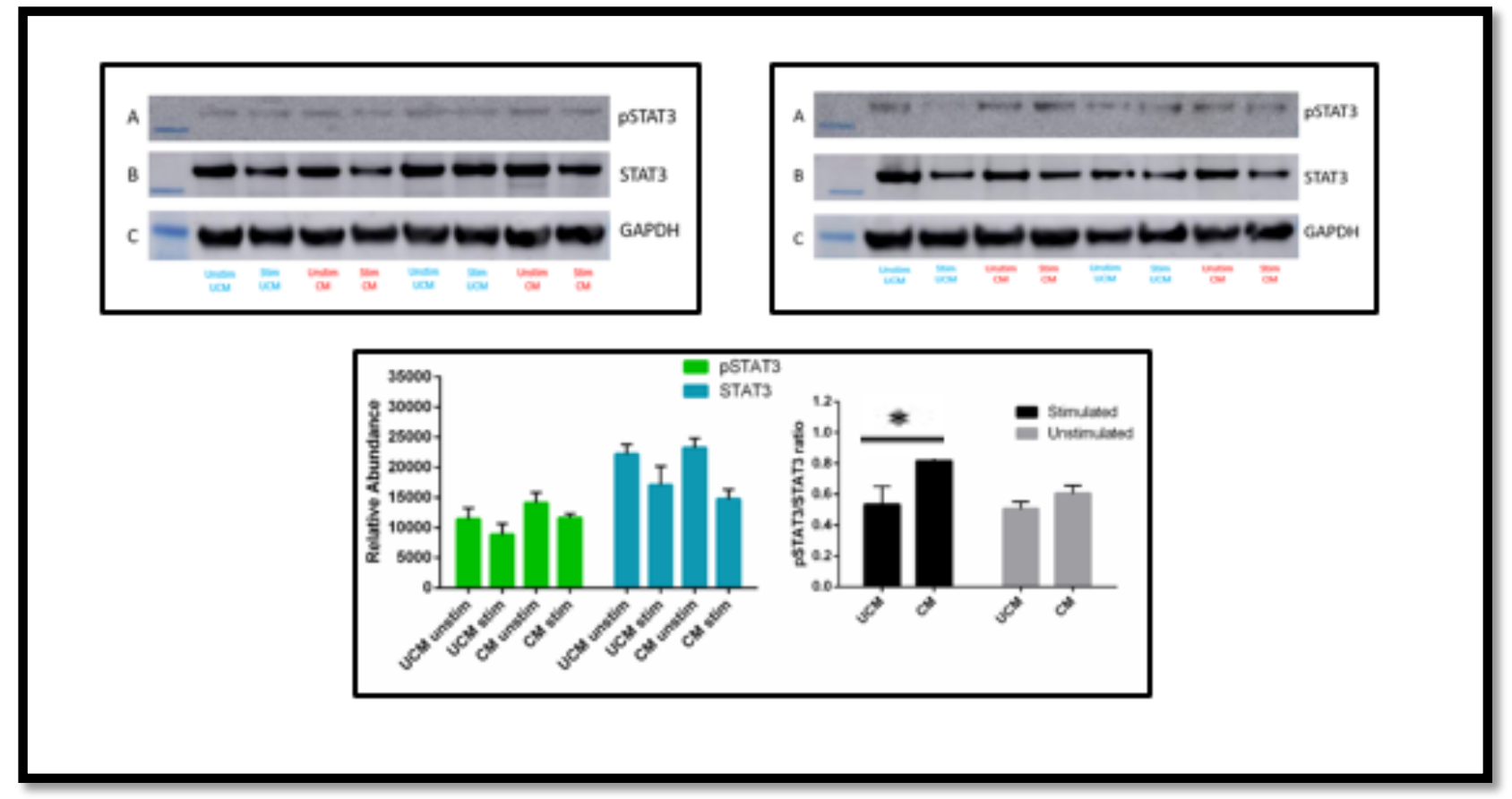

Figure 19: Quantification of pSTAT3 and STAT3 in combined analysis $(n=8)$ for the TG samples. Quantification of the blot showed statistical significance in phosphorylated STAT3 to total STAT3 in the CM stimulated condition. * represents a significant difference with $p<0.05$. 


\section{Chapter 5.}

\section{Discussion}

The muscle wasting associated with cancer cachexia is related to reduced strength and muscle weakness [76]. While a loss of muscle mass is attributed to the severe fatigue experienced by some patients, it has been shown that cancer-induced muscle fatigue can occur without loss of muscle mass [1]. Through this research, we wanted to develop an ex-vivo model that would mirror a cancer-induced model of muscle dysfunction. By achieving this, we intended to study the effects of Interleukin-15 (IL-15) on the induced cachectic conditions in extensor digitorum longus (EDL) muscles and also report the molecular effects of IL-15 on mediators of the JAK-STAT pathway.

Stimulation through exercise is believed to enhance muscle IL-15 secretion $[75,76]$. In fact, secreted IL-15 plays a role as an inflammatory mediator and immune cell chemoattractant and has been reported to be involved in the proliferation, differentiation and activation of NK cells and CD8+ T cells $[77,78]$. Previous work shows IL-15's role extending in promoting a more pro-oxidative phenotype, stimulating heightened fatigue resistance and increasing mitochondrial density $[4,39]$. This mechanism is the reason for using an IL-15TG overexpressing IL-15 mouse model in our current project. It was hypothesized that this additional muscle secreted IL-15 will help attenuate muscle dysfunction that will be seen in this cancer-induced model of muscle dysfunction. We saw no significant differences in muscle function between any of the groups or conditions (Figures 10-13). Therefore, we are inconclusive whether overexpressing IL-15 would potentially rescue muscle function in this model. However, there is evidence suggesting that our exposure time of the cancer cell conditioned media is insufficient for inducing a change in the contractile function of the EDLs. 
This may potentially be the reason why the effect of IL-15 is not clearly exhibited.

Recent studies conducted by Jackman et al. [9] investigated the effect of cancer cellsecreted factors by using mouse colon carcinoma cells (C26) on C2C12 skeletal muscle (SKM) cells. They measured their conditioned media exposed to the SKM cells at different time points for a marker, Leukemia Inhibitory Factor (LIF), which has been identified as the secreted peptide that induces myotube atrophy in a C26 mouse model [31]. The researchers found undetectable LIF concentrations at the 0 hour and 1 hour time points, but did report an increase of LIF at 2 hours after incubation, which increased linearly with time [9]. Similarly, McLean et al. [14] tested Lewis lung carcinoma conditioned medium (LCM) on C2C12 myotubes at different time points to study the effect of LCM cell-secreted factors on mitochondrial electron transport chain function and ROS factor production. They found significance in their proteins of interest at the 2 hour and 24 hour time points, but failed to see an effect at the control and 30 minute time point [14]. This evidence suggests that our basic protocol to study the effects of the MDA-MB-231 cell line secreted factors on muscle function is unfitting as we may not be allowing enough time for the secreted factors to produce an effect on the muscle cells. Nonetheless, it does provide evidence for the continuation of developing and enhancing protocols that might be fitting for this model.

In fact, to test if extended exposure plays a role in our model, we decided to test the last two mice left in both of our TG and TG control colonies, under a modified protocol. Each of the 4 mice were assigned to the 4 different groups (CM TG, UCM TG, CM CON, and UCM CON) and were functionally tested with an additional 2 hour pre-incubation in the respective media. Since no statistical analyses could be performed, due to only having one sample in each group, we decided to plot a graphical representation between the mean of the respective groups with the 
muscle function of the individual samples with extended exposure (Figures 16 and 17). We observe a shift in the EDL fatigue curve to the bottom left in all 4 samples relative to their groups. Similar decrease in Area Under the Fatigue Curve were observed, coinciding with the graphs, for all of the samples, thereby showing a lower total aggregate force. This demonstrates that extended exposure may in fact have a significant effect in our model, both functionally and molecularly. Future studies are needed to build on these data and we will also need to modify the protocol to use extended exposure across all groups and samples.

Another key component of this research that adds novelty to this model is to provide an apt physiological model that studies the muscle as a functional unit instead of using cell culture models. As to our knowledge, all experiments have so far used cell culture models to study pathways and relating them to muscle function. The results represented in Figures 16 and Figure 17 can be used as a testament to this novelty as we see a similar shape of the fatigue curve across all samples, including extended exposure. With this model, we also hope to study the effect of cancer cell-secreted factors on molecular pathways and oxidative stress inducing conditions. Since elevated levels of ROS are seen in mostly all of the different types of cancers and have shown to promote pro-tumorigenic signaling, cancer cell proliferation, and tumor development and progression [64], we decided to conduct experiments assessing the protein carbonyl content using the OxiSelect Protein Carbonyl Fluorometric Assay. We found no evidence of ROS induced carbonylation (Figure 14) and concluded that acute conditioned media exposure does not induce oxidative stress. With regards to measuring ROS induced carbonylation using IL-15TG, overexpressing IL-15 mice it is important to note that IL-15 has been previously investigated as a potential protective mechanism against ROS activity. Li et al. [66] found that pre-incubation with IL-15 reduced the activities of intracellular creatine kinase and lactate dehydrogenase, enzymes 
involved in various metabolic pathways in $\mathrm{C} 2 \mathrm{C} 12$ myoblasts exposed to $\mathrm{H}_{2} \mathrm{O}_{2}$ mediated oxidative stress. We can build on the work done by Li et al. [66] using our model as we can potentially test ROS activity by exposing the muscle as a functional unit to $\mathrm{H}_{2} \mathrm{O}_{2}$ mediated oxidative stress.

Muscle fatigue development has also been attributed to the excess buildup of protons $\left(\mathrm{H}^{+}\right)$in the muscle cells in response to stimulation [74]. A proton buildup in the cell due to the increased glycolytic flux leads to calcium mishandling and aberrant cross-bridge cycling, further causing reduced muscle force and muscle fatigue [70,79]. We decided to measure the $\mathrm{pH}$ of the media prior to starting the protocol and at the end of the protocol in the WT group. However, we saw no significance in the percentage change (Figure 15). In view of the current literature, we attributed our findings to possibly having a small sample size. Perhaps the type of stimulation, which in this case was a low intensity fatigue protocol could have also been a major contributor to our findings. Future work will include $\mathrm{pH}$ measurement in all of the groups to have a larger sample size and will employ high intensity protocols.

Our western blot analyses comparing the level of phosphorylated STAT3 to total STAT3 had exciting results. Western blot analyses were performed on WT and TG samples exposed to the different conditions (CM stimulated, CM unstimulated, UCM stimulated, and UCM unstimulated). Figure 18 represents the combined western blot analysis for all of the samples in the WT group. No statistical significance was seen in any of the groups. However, we saw an opposing result in the western blot analyses of the TG group samples, represented in Figure 19. A statistical significant difference $(\mathrm{p}<0.05)$ was observed in the phosphorylated STAT3 to total STAT3 in the stimulated CM in comparison to the stimulated UCM condition. These data suggest that samples that undergo stimulation and are exposed to $\mathrm{CM}$ are showing a higher significance of phosphorylation of STAT3 that corresponds to increased activation of STAT3. 
While an increased phosphorylation of STAT3 due to exposure to CM is supported by the literature, we observed an increased phosphorylation of STAT3 only in the TG group and not in the WT group. This is crucial since it validates the overexpressing properties of the IL-15TG mouse, since IL-15 binding is known to induce and activate the JAK3/STAT3 pathway, specifically induce phosphorylation of STAT3.

Overall, this research work presented with limitations that need to be addressed to improve the model for future work. As discussed, acute exposure may be unfitting for the purpose of this project. It is fair to assume that muscle dysfunction will correlate with molecular signaling after extended exposure to conditioned media containing cancer cell-secreted factors. Another limitation seen in this project is our control media. Despite plating the UCM in identical conditions as the CM (same amount of DMEM, incubation time, temperature and localization in the same incubator) we believe that media used to grow a non-cancer cell line will serve as a better control since the cells would deplete glucose and other nutrients in the DMEM, thus minimizing any confounding bias.

Although we did not show any differences in the EDLs exposed to the different media types or stimulation conditions, we did establish a working model that could be used to further the understanding of mechanisms in cancer cachexia. We believe that this model is a novel way to test the effects of cancer cell-secreted factors and secretions on muscle function as a whole functioning unit. The use of different mice models including the IL-15TG, overexpressor of IL15 , is useful to evaluate the multidimensional role of cytokine and in this case, the role of IL-15. While the immediate need is to establish significant data using the modified protocol with extended exposure, this model has translational potential as it simulates an in-vivo environment analogous to tumor-bearing animals. With that in mind, using conditioned media from various 
cancer cell lines can be investigated on multiple signaling pathways that have abnormal activation in different diseases states. In conclusion, the research provides support for the sound methodology of this ex-vivo model developed for muscle exposure to tumor cell conditioned media. 


\section{CHAPTER 6.}

\section{References}

[1] M. J. Tisdale, “Mechanisms of cancer cachexia.," Physiol. Rev., vol. 89, no. 2, pp. 381410, 2009.

[2] X. Zhou et al., "Reversal of cancer cachexia and muscle wasting by ActRIIB antagonism leads to prolonged survival," Cell, vol. 142, no. 4, pp. 531-543, 2010.

[3] E. E. Pistilli, G. Guo, and W. T. Stauber, "IL-15R $\alpha$ deficiency leads to mitochondrial and myofiber differences in fast mouse muscles," Cytokine, vol. 61, no. 1, pp. 41-45, 2013.

[4] E. E. Pistilli et al., "Loss of IL-15 receptor alpha alters the endurance, fatigability, and metabolic characteristics of mouse fast skeletal muscles," J Clin Invest, vol. 121, no. 8, pp. 3120-3132, 2011.

[5] A. Quintás-Cardama and S. Verstovsek, "Molecular pathways: JAK/STAT pathway: Mutations, inhibitors, and resistance," Clinical Cancer Research, vol. 19, no. 8. pp. 1933$1940,2013$.

[6] M. Buchert, C. J. Burns, and M. Ernst, "Targeting JAK kinase in solid tumors: Emerging opportunities and challenges," Oncogene, vol. 35, no. 8. pp. 939-951, 2016.

[7] S. J. Thomas, J. A. Snowden, M. P. Zeidler, and S. J. Danson, "The role of JAK/STAT signalling in the pathogenesis, prognosis and treatment of solid tumours," Br. J. Cancer, vol. 113 , no. 3 , pp. 365-371, 2015.

[8] K. A. S. Silva et al., "Inhibition of Stat3 activation suppresses caspase-3 and the ubiquitinproteasome system, leading to preservation of muscle mass in cancer cachexia," J. Biol. 
Chem., vol. 290, no. 17, pp. 11177-11187, 2015.

[9] R. W. Jackman et al., "Continuous release of tumor-derived factors improves the modeling of cachexia in muscle cell culture," Front. Physiol., vol. 8, no. SEP, 2017.

[10] N. P. Gullett, V. C. Mazurak, G. Hebbar, and T. R. Ziegler, "Nutritional Interventions for Cancer-Induced Cachexia," Curr. Probl. Cancer, vol. 35, no. 2, pp. 58-90, 2011.

[11] B. H. L. Tan and K. C. H. Fearon, "Cachexia: Prevalence and impact in medicine," Current Opinion in Clinical Nutrition and Metabolic Care, vol. 11, no. 4. pp. 400-407, 2008.

[12] T. A. Waldmann, "JAK/STAT pathway directed therapy of T-cell leukemia/lymphoma: Inspired by functional and structural genomics," Molecular and Cellular Endocrinology, vol. 451. pp. 66-70, 2017.

[13] V. E. Baracos, T. Reiman, M. Mourtzakis, I. Gioulbasanis, and S. Antoun, "Body composition in patients with non-small cell lung cancer: a contemporary view of cancer cachexia with the use of computed tomography image analysis.," Am. J. Clin. Nutr., vol. 91, no. 4, p. 1133S-1137S, 2010.

[14] J. B. McLean, J. S. Moylan, and F. H. Andrade, "Mitochondria dysfunction in lung cancer-induced muscle wasting in C2C12 myotubes," Front. Physiol., vol. 5, no. DEC, 2014.

[15] W. J. Evans et al., "Cachexia: A new definition,” Clin. Nutr., vol. 27, no. 6, pp. 793-799, 2008.

[16] M. J. Tisdale, "Reversing cachexia,” Cell, vol. 142, no. 4. pp. 511-512, 2010.

[17] Y. Miyamoto, D. L. Hanna, W. Zhang, H. Baba, and H.-J. Lenz, "Molecular Pathways: Cachexia Signaling-A Targeted Approach to Cancer Treatment," Statew. Agric. L. Use 
Baseline 2015, vol. 1, pp. 1-6, 2015.

[18] K. R. Bohnert, Y. S. Gallot, S. Sato, G. Xiong, S. M. Hindi, and A. Kumar, "Inhibition of ER stress and unfolding protein response pathways causes skeletal muscle wasting during cancer cachexia," FASEB J., vol. 30, no. 9, pp. 3053-3068, 2016.

[19] M. H. Glickman and A. Ciechanover, "The Ubiquitin-Proteasome Proteolytic Pathway: Destruction for the Sake of Construction," Physiol. Rev., vol. 82, no. 2, pp. 373-428, 2002.

[20] J. S. Miller et al., "A First-in-Human Phase 1 Study of Subcutaneous Outpatient Recombinant Human IL-15 (rhIL-15) in Adults with Advanced Solid Tumors," Clin. Cancer Res., p. clincanres.2451.2017, 2017.

[21] A. Mishra, L. Sullivan, and M. A. Caligiuri, "Molecular pathways: Interleukin-15 signaling in health and in cancer," Clin. Cancer Res., vol. 20, no. 8, pp. 2044-2050, 2014.

[22] F. Penna, S. Busquets, and J. M. Argilés, "Experimental cancer cachexia: Evolving strategies for getting closer to the human scenario," Semin. Cell Dev. Biol., vol. 54, pp. 20-27, 2016.

[23] D. L. Holliday and V. Speirs, "Choosing the right cell line for breast cancer research.," Breast Cancer Res., vol. 13, p. 215, 2011.

[24] R. Cailleau, R. Young, M. Olivé, and W. J. Reeves, "Breast tumor cell lines from pleural effusions," J. Natl. Cancer Inst., vol. 53, no. 3, pp. 661-674, 1974.

[25] J. Mladkova, M. Sanda, E. Matouskova, and I. Selicharova, "Phenotyping breast cancer cell lines EM-G3, HCC1937, MCF7 and MDA-MB-231 using 2-D electrophoresis and affinity chromatography for glutathione-binding proteins," BMC Cancer, vol. 10, 2010.

[26] H. Rochefort, M. Glondu, M. E. Sahla, N. Platet, and M. Garcia, "How to target estrogen 
receptor-negative breast cancer?," in Endocrine-Related Cancer, 2003, vol. 10, no. 2, pp. 261-266.

[27] J. E. Price, a Polyzos, R. D. Zhang, and L. M. Daniels, "Tumorigenicity and metastasis of human breast carcinoma cell lines in nude mice.," Cancer Res., vol. 50, no. 3, pp. 717 $721,1990$.

[28] M. J. Puppa, S. Gao, and J. A. Carson, "Skeletal muscle glycoprotein $130 \hat{a}^{\mathrm{TM}}$ s role in Lewis lung carcinoma - induced cachexia IL-6 overexpression IL-6r Ab administration," vol. 1, pp. 1-13.

[29] U. a White and J. M. Stephens, "The gp130 receptor cytokine family: regulators of adipocyte development and function.," Curr. Pharm. Des., vol. 17, no. 4, pp. 340-6, 2011.

[30] W. Zhang, N. Duan, T. Song, Z. Li, C. Zhang, and X. Chen, "The emerging roles of forkhead box (FOX) proteins in osteosarcoma," J. Cancer, vol. 8, no. 9, pp. 1619-1628, 2017.

[31] D. N. Seto, S. C. Kandarian, and R. W. Jackman, "A Key Role for Leukemia Inhibitory Factor in C26 Cancer Cachexia.,” J. Biol. Chem., vol. 290, no. 32, pp. 19976-86, 2015.

[32] T. Fukawa et al., "Excessive fatty acid oxidation induces muscle atrophy in cancer cachexia," Nat. Med., vol. 22, no. 6, pp. 666-671, 2016.

[33] E. F. Castillo and K. S. Schluns, "Regulating the immune system via IL-15 transpresentation," Cytokine, vol. 59, no. 3. pp. 479-490, 2012.

[34] C. T. Lutz and L. B. S. Quinn, "Sarcopenia, obesity, and natural killer cell immune senescence in aging: Altered cytokine levels as a common mechanism," Aging, vol. 4, no. 8. pp. 535-546, 2012.

[35] B. Alvarez et al., "Effects of interleukin-15 (IL-15) on adipose tissue mass in rodent 
obesity models: Evidence for direct IL-15 action on adipose tissue," Biochim. Biophys. Acta - Gen. Subj., vol. 1570, no. 1, pp. 33-37, 2002.

[36] J. M. Argilés, F. J. López-Soriano, and S. Busquets, "Therapeutic potential of interleukin15: a myokine involved in muscle wasting and adiposity," Drug Discovery Today, vol. 14, no. 3-4. pp. 208-213, 2009.

[37] L. S. Quinn and B. G. Anderson, "Interleukin-15, IL-15 receptor-alpha, and obesity: Concordance of laboratory animal and human genetic studies," Journal of Obesity, vol. 2011. 2011.

[38] C. Bergamaschi et al., "Secretion and biological activity of short signal peptide IL-15 is chaperoned by IL-15 receptor alpha in vivo.," J. Immunol., vol. 183, no. 5, pp. 3064-72, 2009.

[39] L. S. Quinn, B. G. Anderson, J. D. Conner, and T. Wolden-Hanson, "IL-15 overexpression promotes endurance, oxidative energy metabolism, and muscle PPAR?? SIRT1, PGC-1?? and PGC-1 ?? expression in male mice," Endocrinology, vol. 154, no. 1, pp. 232-245, 2013.

[40] J. E. Krolopp, S. M. Thornton, and M. J. Abbott, "IL-15 activates the Jak3/STAT3 signaling pathway to mediate glucose uptake in skeletal muscle cells," Front. Physiol., vol. 7, no. DEC, 2016.

[41] J. Ye, "Beneficial metabolic activities of inflammatory cytokine interleukin 15 in obesity and type 2 diabetes," Frontiers of Medicine, vol. 9, no. 2. pp. 139-145, 2015.

[42] J. J. O'Shea, S. M. Holland, and L. M. Staudt, "JAKs and STATs in Immunity, Immunodeficiency, and Cancer,” N. Engl. J. Med., vol. 368, no. 2, pp. 161-170, 2013.

[43] S. Hacein-Bey-Abina et al., "Sustained Correction of X-Linked Severe Combined 
Immunodeficiency by ex Vivo Gene Therapy," N. Engl. J. Med., vol. 346, no. 16, pp. 1185-1193, 2002.

[44] J. J. O’Shea, D. M. Schwartz, A. V. Villarino, M. Gadina, I. B. McInnes, and A. Laurence, “The JAK-STAT Pathway: Impact on Human Disease and Therapeutic Intervention," Annu. Rev. Med., vol. 66, no. 1, pp. 311-328, 2015.

[45] M. A. Frias and C. Montessuit, "JAK-STAT signaling and myocardial glucose metabolism.," Jak-Stat, vol. 2, no. 4, p. e26458, 2013.

[46] A. Bonetto et al., "STAT3 activation in skeletal muscle links muscle wasting and the acute phase response in cancer cachexia," PLoS One, vol. 6, no. 7, 2011.

[47] H. Baumann and J. Gauldie, "The acute phase response.," Immunol. Today, vol. 15, no. 2, pp. 74-80, 1994.

[48] I. Kushner, "Regulation of the Acute Phase Response by Cytokines," Perspect. Biol. Med., vol. 36, no. 4, pp. 611-622, 1993.

[49] M. J. Tisdale, “Cachexia in cancer patients," Nature Reviews Cancer, vol. 2, no. 11. pp. $862-871,2002$.

[50] J. S. Falconer et al., "Acute-phase protein response and survival duration of patients with pancreatic cancer.," Cancer, vol. 75, pp. 2077-2082, 1995.

[51] N. A. Stephens, R. J. E. Skipworth, and K. C. H. Fearon, "Cachexia, survival and the acute phase response,” Curr. Opin. Support. Palliat. Care, vol. 2, no. 4, pp. 267-274, 2008.

[52] T. A. Zimmers, M. L. Fishel, and A. Bonetto, "STAT3 in the systemic inflammation of cancer cachexia.," Semin. Cell Dev. Biol., vol. 54, pp. 28-41, 2016.

[53] G.-Y. Liou and P. Storz, "Reactive oxygen species in cancer," Free Radic. Res., vol. 44, 
no. 5, pp. 479-496, 2010.

[54] P. D. Ray, B.-W. Huang, and Y. Tsuji, "Reactive oxygen species (ROS) homeostasis and redox regulation in cellular signaling," Cell. Signal., vol. 24, no. 5, pp. 981-990, 2012.

[55] T. B. Dansen and K. W. A. Wirtz, "The peroxisome in oxidative stress," IUBMB Life, vol. 51, no. 4. pp. 223-230, 2001.

[56] R. Liang and S. Ghaffari, "Stem Cells, Redox Signaling, and Stem Cell Aging," Antioxid. Redox Signal., vol. 20, no. 12, pp. 1902-1916, 2014.

[57] J. Martinez-Useros, W. Li, M. Cabeza-Morales, and J. Garcia-Foncillas, “Oxidative Stress: A New Target for Pancreatic Cancer Prognosis and Treatment," J. Clin. Med., vol. 6, no. 3, p. 29, 2017.

[58] M. Nita and A. Grzybowski, "The Role of the Reactive Oxygen Species and Oxidative Stress in the Pathomechanism of the Age-Related Ocular Diseases and Other Pathologies of the Anterior and Posterior Eye Segments in Adults," Oxidative Medicine and Cellular Longevity, vol. 2016. 2016.

[59] G. Barrera, "Oxidative Stress and Lipid Peroxidation Products in Cancer Progression and Therapy," ISRN Oncol., vol. 2012, pp. 1-21, 2012.

[60] Y. J. Suzuki, M. Carini, and D. A. Butterfield, "Protein Carbonylation," Antioxid. Redox Signal., vol. 12, no. 3, pp. 323-325, 2010.

[61] M. Fedorova, R. C. Bollineni, and R. Hoffmann, "Protein carbonylation as a major hallmark of oxidative damage: Update of analytical strategies," Mass Spectrometry Reviews, vol. 33, no. 2. pp. 79-97, 2014.

[62] X. Li, P. Fang, J. Mai, E. T. Choi, H. Wang, and X. F. Yang, "Targeting mitochondrial reactive oxygen species as novel therapy for inflammatory diseases and cancers," Journal 
of Hematology \& Oncology, p. 19, 2013.

[63] D. Weber, M. J. Davies, and T. Grune, "Determination of protein carbonyls in plasma, cell extracts, tissue homogenates, isolated proteins: Focus on sample preparation and derivatization conditions," Redox Biology, vol. 5. pp. 367-380, 2015.

[64] K. Ishikawa et al., "ROS-generating mitochondrial DNA mutations can regulate tumor cell metastasis.," Science, vol. 320, no. 5876, pp. 661-4, 2008.

[65] C. R. Reczek and N. S. Chandel, "The Two Faces of Reactive Oxygen Species in Cancer," Annu. Rev. Cancer Biol., vol. 1, no. 1, pp. 79-98, 2017.

[66] F. Li et al., "Protective effect of myokine IL-15 against $\mathrm{H} 2 \mathrm{O} 2$-mediated oxidative stress in skeletal muscle cells.," Mol. Biol. Rep., vol. 41, no. 11, pp. 7715-22, 2014.

[67] L. S. Quinn, B. G. Anderson, L. Strait-Bodey, A. M. Stroud, and J. M. Argiles, "Oversecretion of interleukin-15 from skeletal muscle reduces adiposity," AJP Endocrinol. Metab., vol. 296, no. 1, pp. E191-E202, 2008.

[68] A. Pasut, A. E. Jones, and M. A. Rudnicki, "Isolation and culture of individual myofibers and their satellite cells from adult skeletal muscle.," J. Vis. Exp., no. 73, p. e50074, 2013.

[69] CellBiolabs, "OxiSelect ROS Assay kit," OxiSelect ROS Assay kit, 2009.

[70] S. C. Bodine et al., "Identification of ubiquitin ligases required for skeletal Muscle Atrophy,” Science (80-. )., vol. 294, no. 5547, pp. 1704-1708, 2001.

[71] H. N. Langstein and J. a Norton, "Mechanisms of cancer cachexia.," Hematol. Oncol. Clin. North Am., vol. 5, no. 1, pp. 103-123, 1991.

[72] D. G. Allen, H. Westerblad, and J. Lannergren, "The role of intracellular acidosis in muscle fatigue," Adv Exp Med Biol, vol. 384, pp. 57-68, 1995.

[73] P. B. CHASE and M. J. KUSHMERICK, "EFFECTS OF PH ON CONTRACTION OF 
RABBIT FAST AND SLOW SKELETAL-MUSCLE FIBERS,” Biophys. J., vol. 53, no. 6, pp. 935-946, 1988.

[74] S. K. Stackhouse, D. S. Reisman, and S. A. Binder-Macleod, "Challenging the Role of pH in Skeletal Muscle Fatigue," Phys. Ther., vol. 81, no. 12, pp. 1897-1903, 2001.

[75] R. H. Fitts, "The role of acidosis in fatigue: Pro perspective," Medicine and Science in Sports and Exercise, vol. 48, no. 11. pp. 2335-2338, 2016.

[76] Y. Guo, F. Xu, T. Lu, Z. Duan, and Z. Zhang, "Interleukin-6 signaling pathway in targeted therapy for cancer," Cancer Treatment Reviews, vol. 38, no. 7. pp. 904-910, 2012.

[77] X. Zhang, S. Sun, I. Hwang, D. F. Tough, and J. Sprent, "Potent and selective stimulation of memory-phenotype CD8+ T cells in vivo by IL-15.," Immunity, vol. 8, no. 5, pp. 5919, 1998.

[78] Y. Tagaya et al., "Generation of secretable and nonsecretable interleukin 15 isoforms through alternate usage of signal peptides.," Proc. Natl. Acad. Sci. U. S. A., vol. 94, no. 26, pp. 14444-14449, 1997.

[79] T. A. Waldmann and Y. Tagaya, “THE MULTIFACETED REGULATION OF INTERLEUKIN-15 EXPRESSION AND THE ROLE OF THIS CYTOKINE IN NK CELL DIFFERENTIATION AND HOST RESPONSE TO INTRACELLULAR PATHOGENS," Annu. Rev. Immunol., vol. 17, no. 1, pp. 19-49, 1999.

[80] J. P. Lodolce et al., "IL-15 receptor maintains lymphoid homeostasis by supporting lymphocyte homing and proliferation," Immunity, vol. 9, no. 5, pp. 669-676, 1998.

[81] A. J. McComas, In skeletal muscle form and function. 1996. 


\section{CHAPTER 7.}

\section{Supplementary Data}

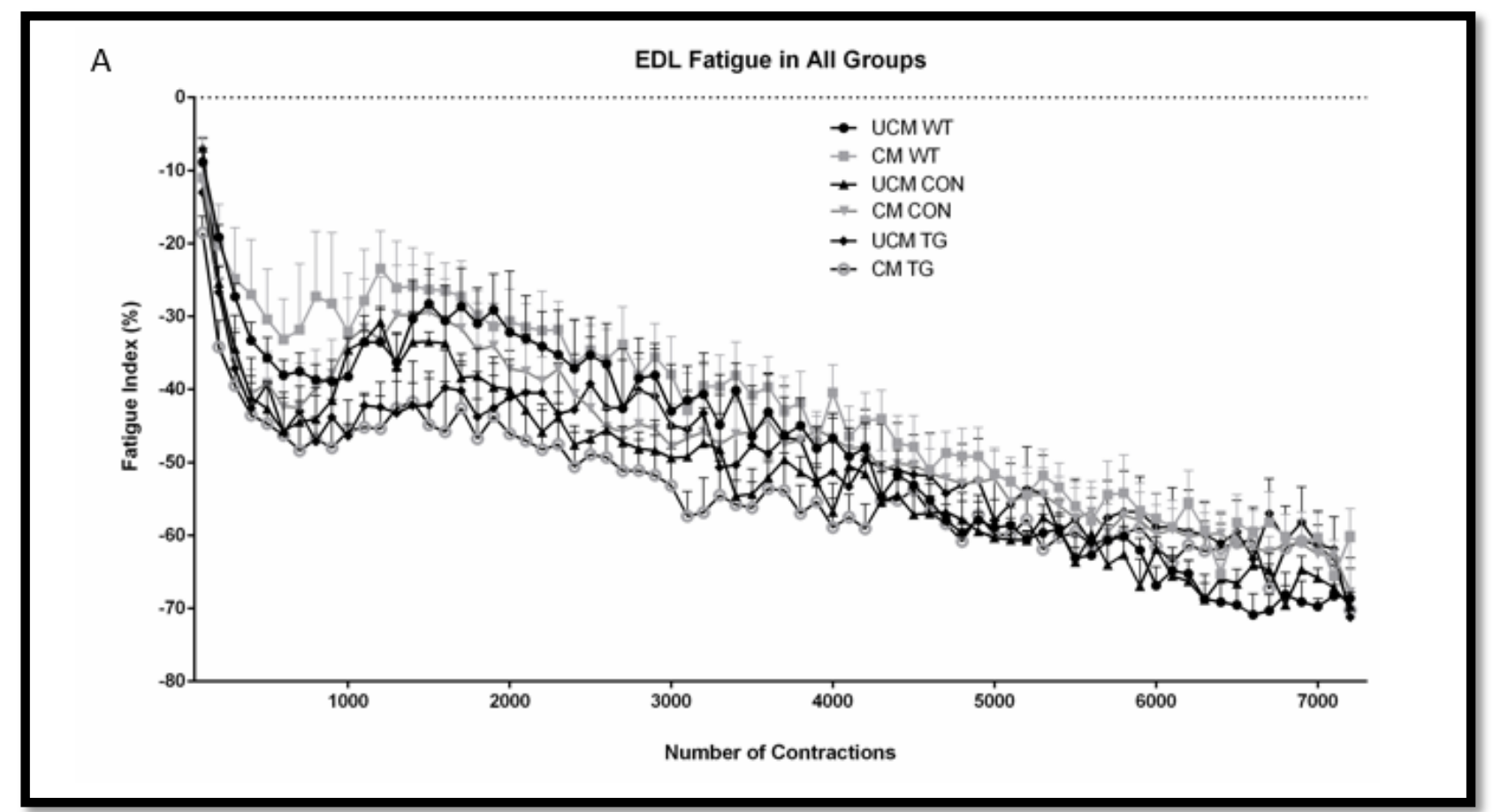

Supplementary Figure 1: EDL Fatigue curve for all groups.

Figure S1 shows the EDL fatigue curves for all the conditions (UCM WT, CM WT, UCM TG,CM $T G$, UCM CON, and CM CON). No statistical differences were seen between any of the groups. 


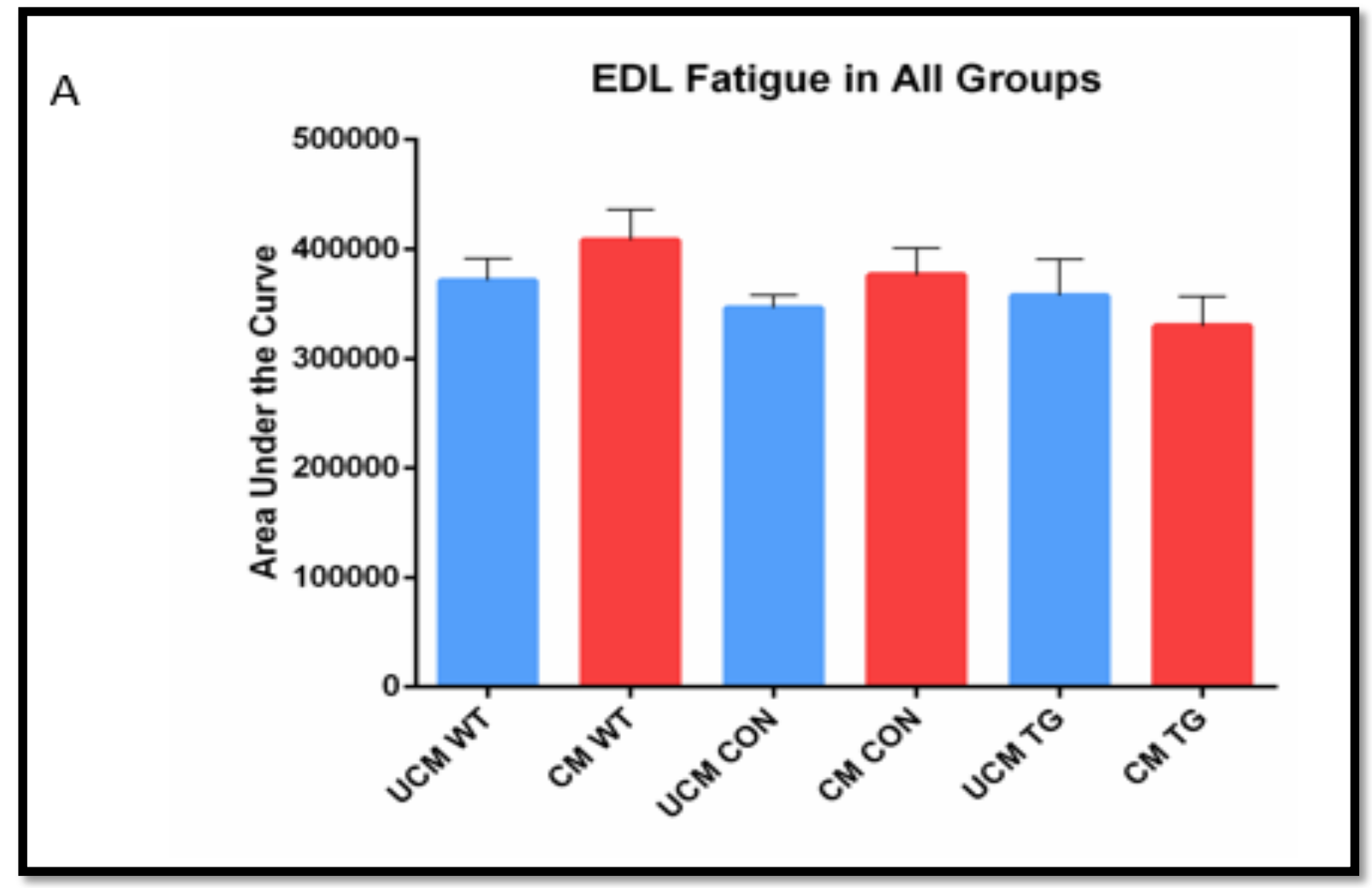

Supplementary Figure 2: EDL Area Under the Fatigue Curve for all groups.

Figure $S 2$ shows the EDL Total Area Under the Fatigue Curves $\left(m N^{*} S\right)$ for all the conditions (UCM WT, CM WT, UCM TG, CM TG, UCM CON, and CM CON). No statistical differences were seen between any of the groups. 
A

\section{Carbonyl Concentration in All Groups}

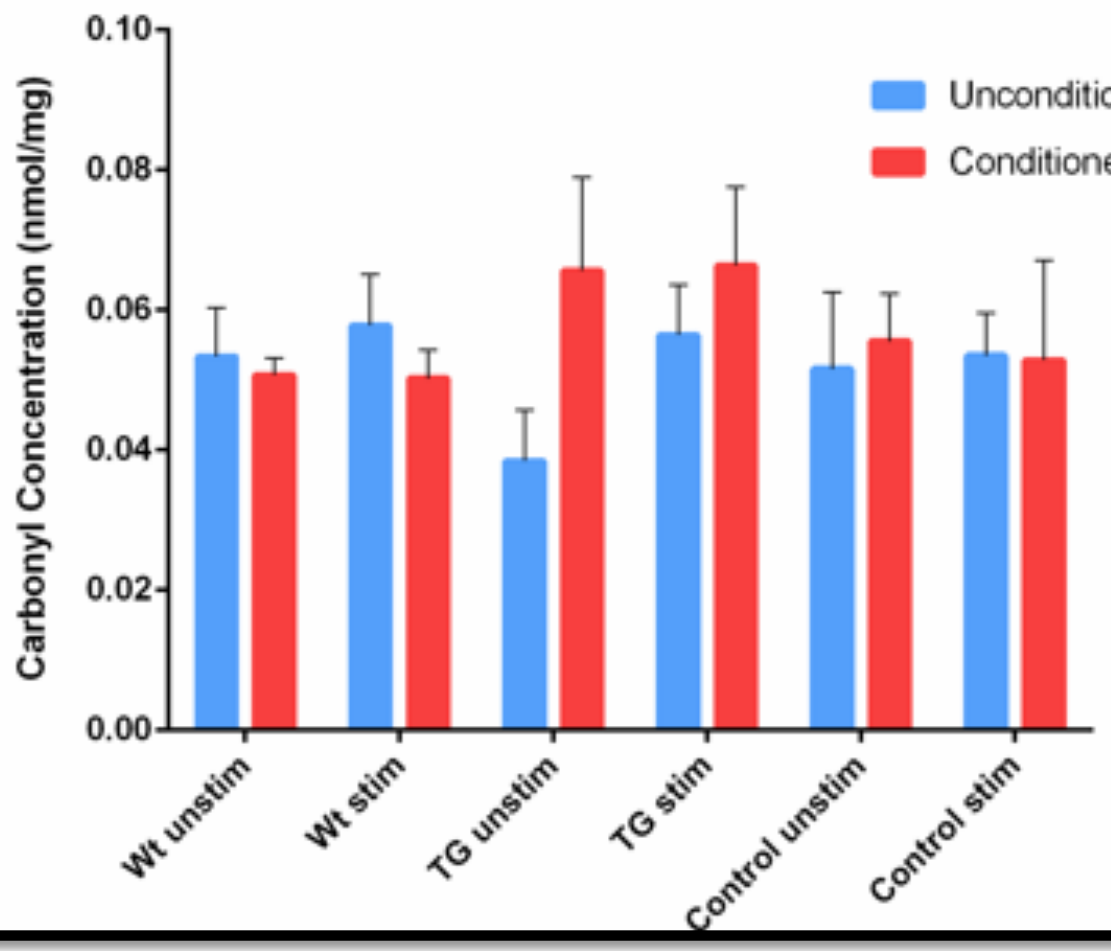

Supplementary Figure 3: Carbonyl Concentration (nmol/mg) in all groups.

Figure S3 shows the Carbonyl concentrations present in the different groups and conditions (UCM \& CM WT stimulated, UCM \& CM WT unstimulated, UCM \& CM TG stimulated, UCM \& CM TG unstimulated, UCM \& CM CON stimulated, UCM \& CM CON unstimulated). No statistical differences were seen between any of the groups. 


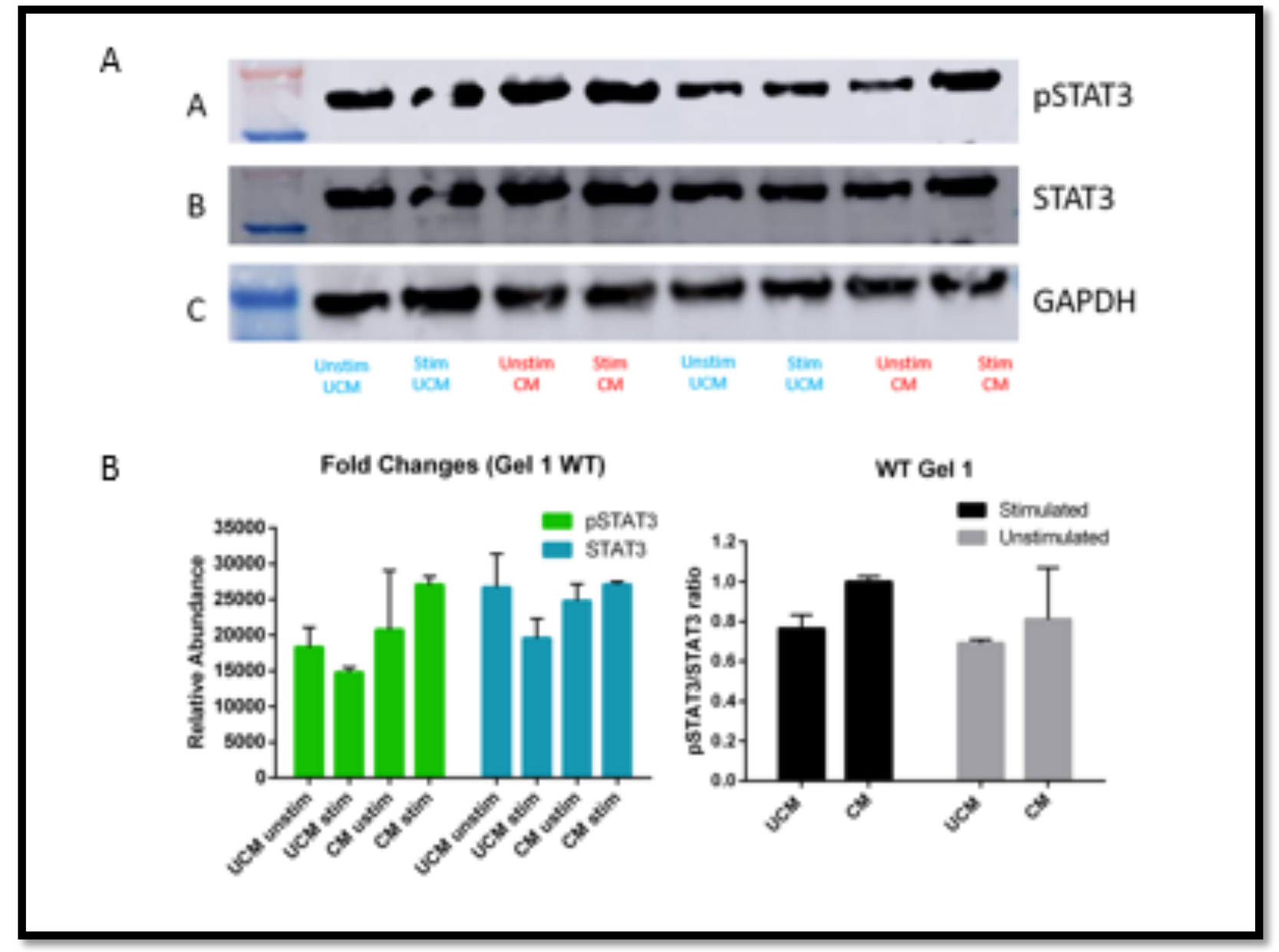

Supplementary Figure 4: Quantification of pSTAT3 and STAT3 in gel $1(n=8)$ for the WT samples.

Panel $\boldsymbol{A}$ shows the western blot corresponding to gel 1 of the WT samples. Panel $\boldsymbol{B}$ shows the quantification of phosphorylated STAT3 to total STAT3. No statistical differences were seen between any of the groups. 


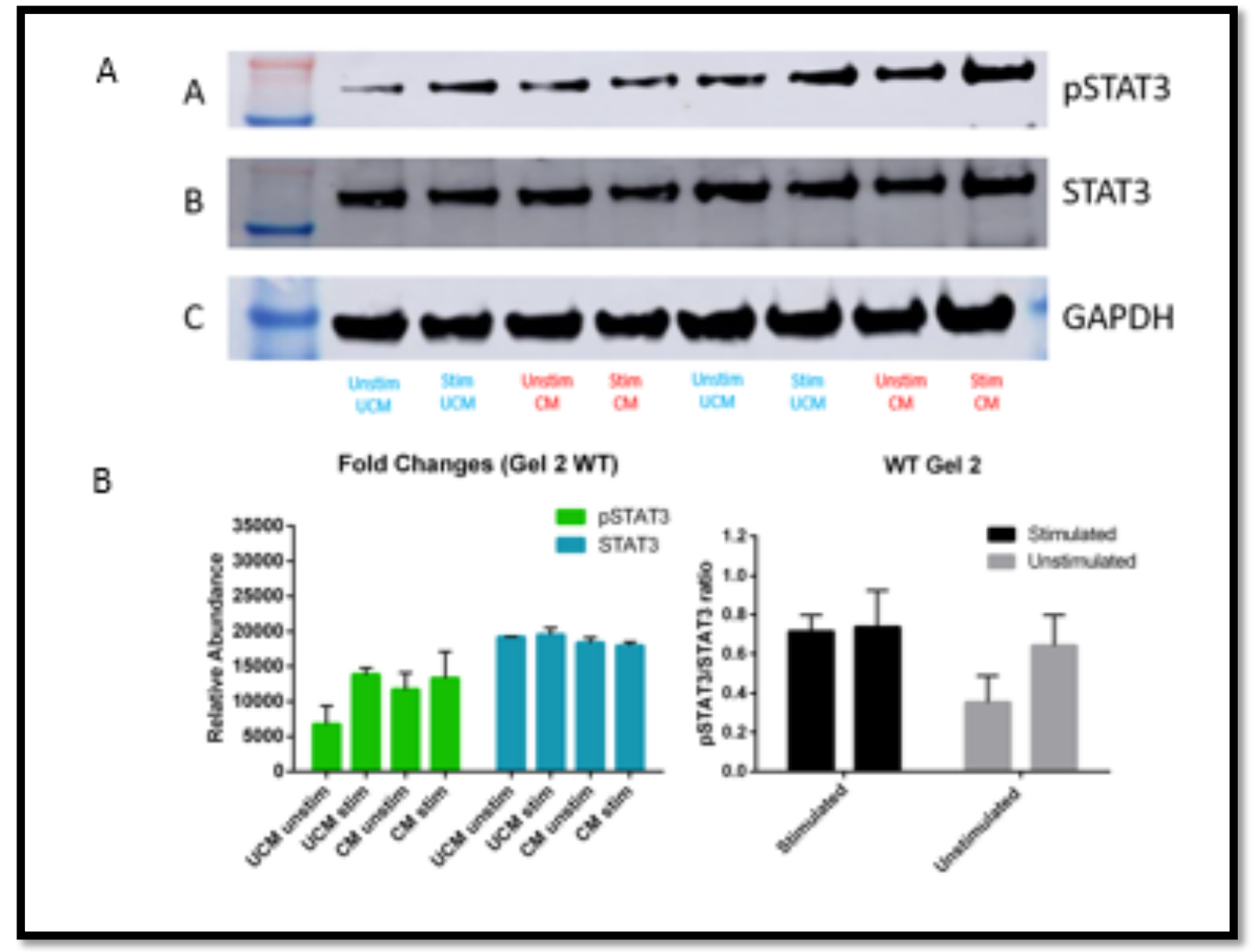

Supplementary Figure 5: Quantification of pSTAT3 and STAT3 in gel $2(n=8)$ for the WT samples.

Panel $\boldsymbol{A}$ shows the western blot corresponding to gel 2 of the WT samples. Panel $\boldsymbol{B}$ shows the quantification of phosphorylated STAT3 to total STAT3. No statistical differences were seen between any of the groups. 


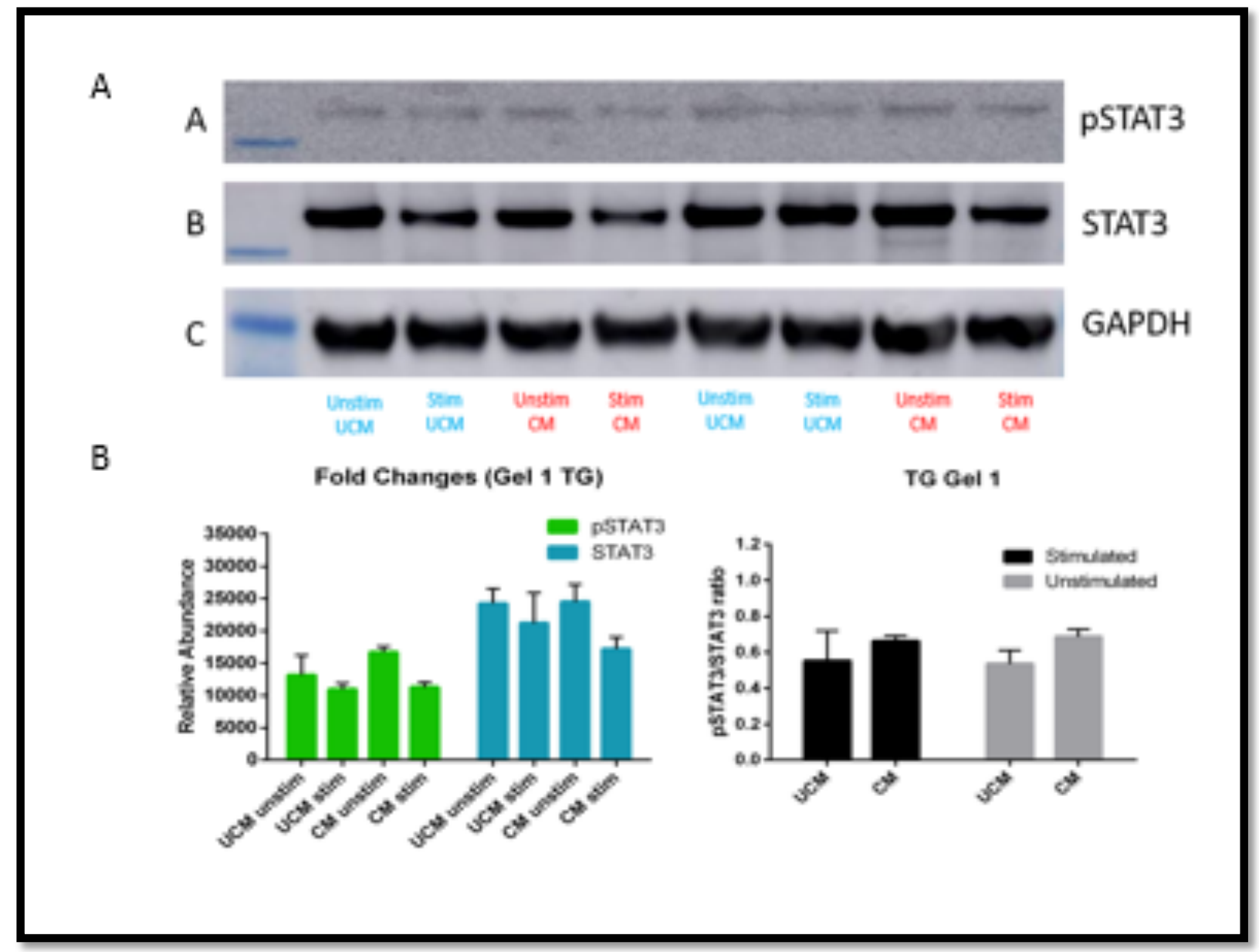

Supplementary Figure 6: Quantification of pSTAT3 and STAT3 in gel $1(n=8)$ for the TG samples.

Panel $\boldsymbol{A}$ shows the western blot corresponding to gel 1 of the TG samples. Panel B shows the quantification of phosphorylated STAT3 to total STAT3. No statistical differences were seen between any of the groups. 
A

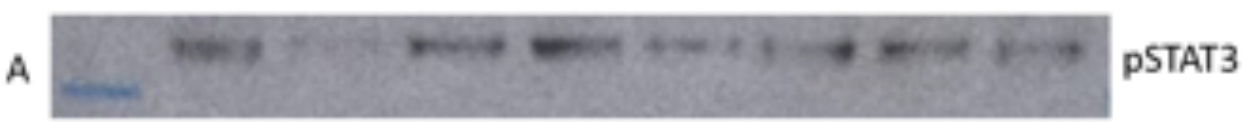

B

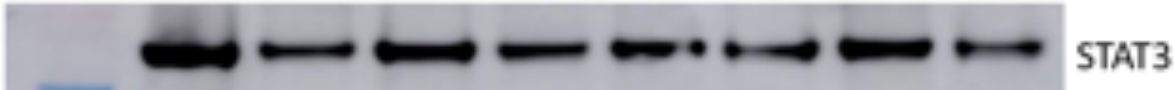

C

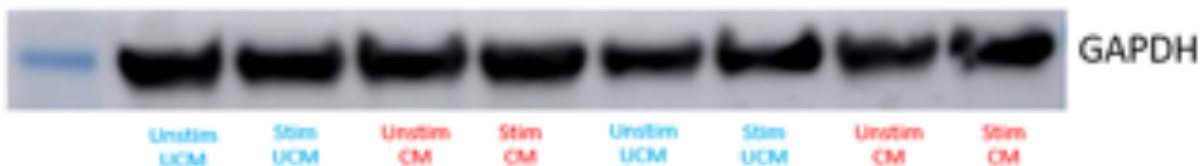

B

Fold Changes (Gel 2 TG)

TG Gel 2
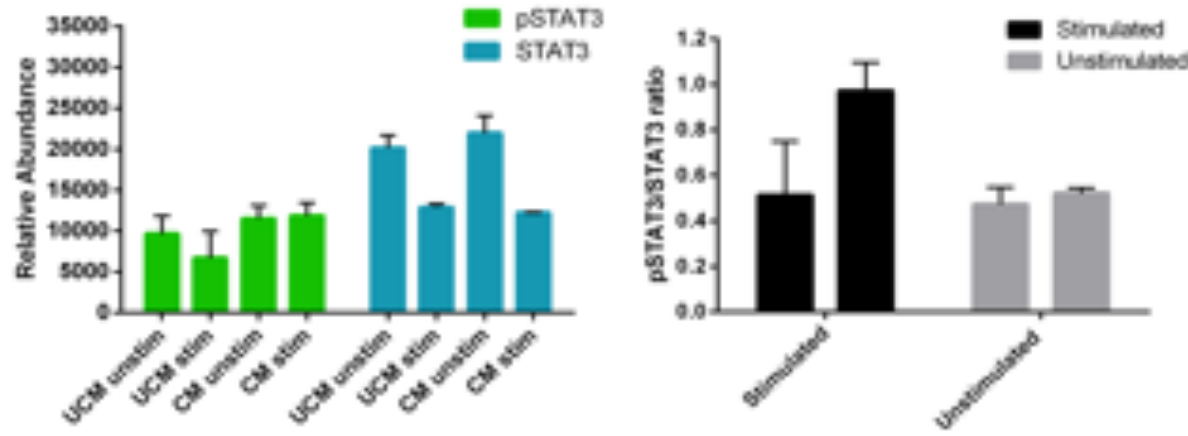

Supplementary Figure 7: Quantification of pSTAT3 and STAT3 in gel $2(n=8)$ for the TG samples.

Panel $\boldsymbol{A}$ shows the western blot corresponding to gel 2 of the TG samples. Panel B shows the quantification of phosphorylated STAT3 to total STAT3. No statistical differences were seen between any of the groups. 